\title{
Developmentally regulated activation of defense allows for rapid inhibition of infection in age-related resistance to Phytophthora capsici in cucumber fruit
}

\author{
Ben N. Mansfeld ${ }^{1}$, Marivi Colle ${ }^{1}$, Chunqiu Zhang ${ }^{1,2}$, Ying-Chen Lin $^{1}$ and Rebecca Grumet ${ }^{1 *}$ (D)
}

\begin{abstract}
Background: Age-related resistance (ARR) is a developmentally regulated phenomenon conferring resistance to pathogens or pests. Although ARR has been observed in several host-pathogen systems, the underlying mechanisms are largely uncharacterized. In cucumber, rapidly growing fruit are highly susceptible to Phytophthora capsici but become resistant as they complete exponential growth. We previously demonstrated that ARR is associated with the fruit peel and identified gene expression and metabolomic changes potentially functioning as preformed defenses.

Results: Here, we compare the response to infection in fruit at resistant and susceptible ages using microscopy, quantitative bioassays, and weighted gene co-expression analyses. We observed strong transcriptional changes unique to resistant aged fruit 2-4 h post inoculation (hpi). Microscopy and bioassays confirmed this early response, with evidence of pathogen death and infection failure as early as 4 hpi and cessation of pathogen growth by 8-10 hpi. Expression analyses identified candidate genes involved in conferring the rapid response including those encoding transcription factors, hormone signaling pathways, and defenses such as reactive oxygen species metabolism and phenylpropanoid biosynthesis.

Conclusion: The early pathogen death and rapid defense response in resistant-aged fruit provide insight into potential mechanisms for ARR, implicating both pre-formed biochemical defenses and developmentally regulated capacity for pathogen recognition as key factors shaping age-related resistance.
\end{abstract}

Keywords: Age-related resistance, Ontogenic resistance, Cucumber, Phytophthora capsici, Plant defense, Transcriptome, Co-expression networks

\section{Background}

Ontogenic, developmental, or age-related resistance (ARR), wherein plants or plant organs transition from susceptibility to resistance as a result of developmental changes [1-3], has been described in several different plant-pathogen systems and in crops such as pepper, grape, rice, wheat, and several

\footnotetext{
* Correspondence: grumet@msu.edu

${ }^{1}$ Graduate Program in Plant Breeding, Genetics and Biotechnology and Department of Horticulture, Michigan State University, 1066 Bogue St, East Lansing, MI 48824, USA

Full list of author information is available at the end of the article
}

cucurbit crops [4-8]. While providing protection in agricultural systems and potentially playing an important role in the evolution and optimization of host resistance [9], the molecular mechanisms controlling these resistances are not well understood.

Evidence from various systems suggests possible physical, chemical, or physiological changes that may result from age-dependent, non-pathogen specific investment in defense such as cell wall modifications, production of antimicrobial phytoanticipins, or altered hormone balance $[2,9$, 
10]. There are also some examples where ARR may result from developmentally regulated expression of a pathogen receptor. In rice, a developmental increase in expression of leucine-rich repeat (LRR)-kinase type genes, Xa3/Xa26 and $\mathrm{Xa21}$, that peaks at the maximum-tillering stage of growth, confers ARR to bacterial blight [7, 11]. Recently, in Arabidopsis, transcriptional control of the canonical immune receptor FLS2 was also shown to regulate ontogenic resistance to Pseudomonas syringae [12]. Thus, in these examples, a newly acquired ability to perceive the pathogen allows for an induced resistance response at the resistant age.

One model system for organ specific ARR is cucumber fruit rot by the oomycete Phytophthora capsici [13-15]. This soilborne hemibiotroph is a pathogen of many agriculturally important crops including numerous solanaceous and cucurbit species [16]. Infection is initiated by means of biflagellate zoospores which arrive via water from rain or irrigation [17]. Upon reaching the host target tissue, zoospores encyst, lose their flagella and germinate forming germination tubes [18]. The germination tubes penetrate the plant surface using appressoria and continue growing hyphae. During the early, biotrophic stages of infection, haustoria are formed mediating direct interaction with the host cells and nutrient acquisition. Studies in tomato show that the pathogen then transitions to necrotrophy at approximately $48 \mathrm{~h}$ postinoculation (hpi) and can produce sporangia for asexual reproduction as soon as $72 \mathrm{hpi}[18,19]$. The transcriptome of $P$. capsici infection has been described in tomato leaves using microarray technology [19]. Two major transcriptomic responses were identified: 1 ) at initial infection (8 hpi) at which host primary metabolism was downregulated and specialized metabolism was upregulated, and 2) at the transition to necrotrophy (48 hpi) when large scale transcriptional reprograming occurs in the host [19].

In cucumber, the primary targets of infection of $P$. capsici are the fruit, which display symptoms of rot and tissue collapse followed by appearance of white mycelia and sporangia. Testing of the cucumber germplasm collection found that very young fruit [less than 8 days post pollination (dpp)] from the great majority of cucumber accessions are highly susceptible [20]. The genetics of inheritance of young fruit resistance have not yet been determined, and to date, no stable resistance to this pathogen is deployed in cultivated cucumber. However, an ARR was observed in a few cucumber cultivars and plant introduction lines wherein fruit become resistant as they near the end of their exponential growth phase, at $\sim 12-15$ days post-pollination (dpp) [13]. As with young fruit resistance, the genetics of ARR have not been determined. However, transcriptional analyses indicate that the end of exponential fruit growth is accompanied by downregulation of transcription factors and other genes involved in growth and by increased expression of defense related genes [14, 15, 21]. This transcriptional transition away from growth and towards defense, corresponding with the end of rapid growth, is congruent with current understanding of optimal defense programing and utilization of resources [22]. With growth largely completed, fruit can invest in defense to protect the developing seeds during the period of seed maturation that precedes the final transition to fruit ripening at $\sim 35 \mathrm{dpp}$.

Further studies showed that the cucumber fruit peel is important for ARR [14]. Excised peels exhibit equivalent responses (susceptible or resistant) as do intact whole fruit, and if the peel is wounded or removed from resistant-aged fruit the underlying tissue is highly susceptible [14]. Furthermore, methanolic extracts from resistant-aged peels had inhibitory effects on pathogen growth and cucumber peels of resistant-aged fruit vs. susceptible age are enriched for genes associated with defense against biotic and abiotic stresses [14]. ARR to $P$. capsici also has been observed in whole pepper plants [4], chile pepper fruit [23], and several cucurbit fruits [6, 10]. Similar to cucumber, wounding overcame the resistance in chili pepper fruit and squash. The ARR in pepper and squash was suggested to result from structural changes in fruit surface properties such as cuticle thickness or cell wall lignification $[10,23]$.

Collectively, studies of ARR to date suggest that it may arise by means of preformed and/or induced defenses. In either case, a developmentally regulated difference in expression of the defense components is required. A comparison of uninoculated peel transcriptomes of ARR expressing and non-expressing cucumber fruit revealed the potential for either or both cases [15]. Of the 80 genes that were uniquely upregulated in ARR expressing fruit at the resistant age, four putative resistance genes (R-genes) as well as resistance related transcription factors were identified. Furthermore, this set of genes was highly enriched for specialized metabolism genes, including terpenoid synthesis and decoration genes, and untargeted metabolomic analyses of the same tissues revealed an increased accumulation of glycosylated terpenoids in the resistant tissue [15]. The accumulation of these preformed compounds may work in inhibiting infection, while at the same time developmentally regulated expression of $R$-genes may provide the ontogenic capacity to sense and respond to infection.

A highly resolved transcriptomic characterization of early of infection in ARR response could shed light on the mechanism by which this resistance is controlled, revealing if preformed or induced defenses are recruited. Furthermore, utilizing an ARR pathosystem allows a 
unique opportunity to examine both compatible and incompatible interactions within the same plant genotype. To our knowledge only one other transcriptomic study of ARR was performed, in that case apple leaves of different ages inoculated with Venturia inaequalis were sampled at 72 and 96 hpi [24]. In this study, our goal was to determine whether a developmentally regulated inducible mechanism contributes to ARR of cucumber fruit to $P$. capsici. To address this question, we performed microscopic, quantitative bioassay, and transcriptomic time courses characterizing the response of cucumber peel to inoculation with $P$. capsici at resistant (16 dpp) and susceptible (8 dpp) ages during the first 48 hpi. Differential expression analysis combined with weighted gene-co-expression network analyses and cubic spline regression analysis identified modules and defense-related genes which were uniquely induced in resistant fruit at early timepoints post-inoculation. The findings suggest that a developmentally acquired ability to initiate early defense responses may be crucial in conferring ARR to P. capsici in cucumber.

\section{Results}

The cultivar 'Poinsett 76' displays age-related resistance to $P$. capsici

Our previous ARR studies [6, 13-15] examined 'Vlaspik', an $F_{1}$ hybrid commonly grown for processing cucumber production in the midwestern USA. To facilitate future analyses, we sought to identify a homozygous inbred cultivar expressing ARR. Testing of 22 inbred cucumber cultivars was performed using a detached fruit assay [13]. Hand-pollinated fruit were harvested at 8 and 16 dpp. The unwounded fruit surface was inoculated with droplets of $P$. capsici zoospore suspension and evaluated over a period of ten days using a 9-point disease score rating (Supplementary Table 1). Two of the inbred cultivars were identified to exhibit ARR, including the fresh market cultivar 'Poinsett 76' (Fig. 1). As described for 'Vlaspik', very young 'Poinsett 76 ' fruit are extremely susceptible to infection, showing severe symptoms and extensive mycelial growth and sporulation (Fig. 1 a). Fruits then become increasingly resistant as they complete their exponential growth phase. As fruits reached full size, at $\sim 16 \mathrm{dpp}$, they primarily exhibited localized necrosis at sites of inoculation, with occasional successful infection at inoculation sites (Fig. 1 b).

\section{Age-dependent differential transcriptomic responses to infection}

As a first step to explore the early stages of infection in susceptible age fruit $(8 \mathrm{dpp})$ we observed germination and growth of a constitutively fluorescent isolate of $P$. capsici NY-0644-1 [25]. Peel sections from young fruit were inoculated with droplets of zoospore suspension, prepared for microscopy, and observed for $72 \mathrm{~h}$. Consistent with observations of $P$. capsici development on tomato leaves [19], appressoria formation was observed by $4 \mathrm{hpi}$, extensive growth by $24 \mathrm{hpi}$, and sporangia formation by 72 hpi (Fig. $1 \mathrm{c}$ ).

Based on these results we compared transcriptomic responses of resistant (16 dpp) and susceptible ( $8 \mathrm{dpp})$ fruit peels at 0 (uninoculated), 4, 24, and 48 hpi. For each age and timepoint three fruit were inoculated with 6-12 droplets. All inoculation sites for a given fruit were harvested and pooled for sequencing providing $\sim 20 \mathrm{M}$ reads per sample. An average of $\sim 82 \%$ reads uniquely quasi-mapped to the cucumber transcriptome (Supplementary Figure 1). Pearson's correlations of replicate samples were at least 96\% (Supplementary Figure 2) showing high reproducibility among replicates.

To first identify any age-dependent differences between 8 and $16 \mathrm{dpp}$ fruit, we performed transcriptional comparisons of the uninoculated control fruit peels. This analysis revealed that over 3400 and 4300 genes were up- and down-regulated genes with age, respectively. Gene Ontology (GO) term enrichment analyses (Supplementary Table 2) revealed that "specialized metabolic processes" ( $p$-value $=7.50 \mathrm{e}-05)$ and "defense response to fungus" ( $p$-value $=9.11 \mathrm{e}-03$ ) were among the upregulated terms. These results are consistent with our previous developmental analyses of other cucumber cultivars $[14,15]$ indicating potential differences in pre-formed factors contributing to resistance. Also consistent with prior studies and with the difference in developmental stages of the fruits, the most significantly enriched down-regulated term was "photosynthesis, light reaction" $(p$-value $=1.40 \mathrm{e}-26)$.

Principal component analysis (PCA) of the transcriptome data from inoculated and uninoculated samples showed that the first principal component largely reflected time post inoculation, while the second largely reflected fruit age (Fig. 2). A similar transcriptional shift in direction and magnitude was observed along the positive direction of PC1 at 4 hpi regardless of age (8 dpp - circles, $16 \mathrm{dpp}$ - triangles) of the tissue, suggesting a somewhat comparable initial response to infection (Fig. 2 a). In contrast, subsequent timepoints (colors) exhibited differential transcriptional responses to infection as evidenced by the PCA. The susceptible $8 \mathrm{dpp}$ samples progressively moved along the positive direction of $\mathrm{PC} 1$ with time, while resistant $16 \mathrm{dpp}$ samples largely stayed in same position relative to $\mathrm{PC1}$, suggesting little subsequent change in gene expression. One $16 \mathrm{dpp}$ sample in each of 24 and $48 \mathrm{hpi}$ timepoints exhibited transcription signatures that approached those of infected $8 \mathrm{dpp}$ fruit at the same respective timepoints. As successful infection can occasionally occur on $16 \mathrm{dpp}$ fruit, this was likely the case for those two samples. These samples had little effect on 


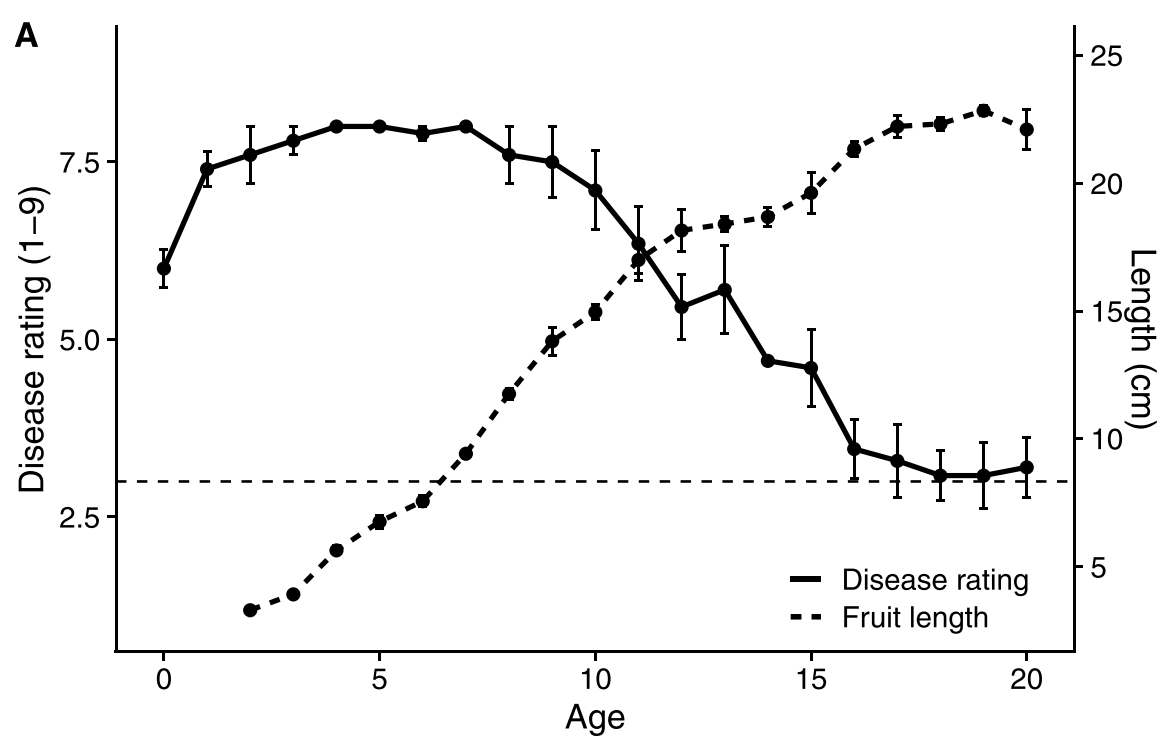

B
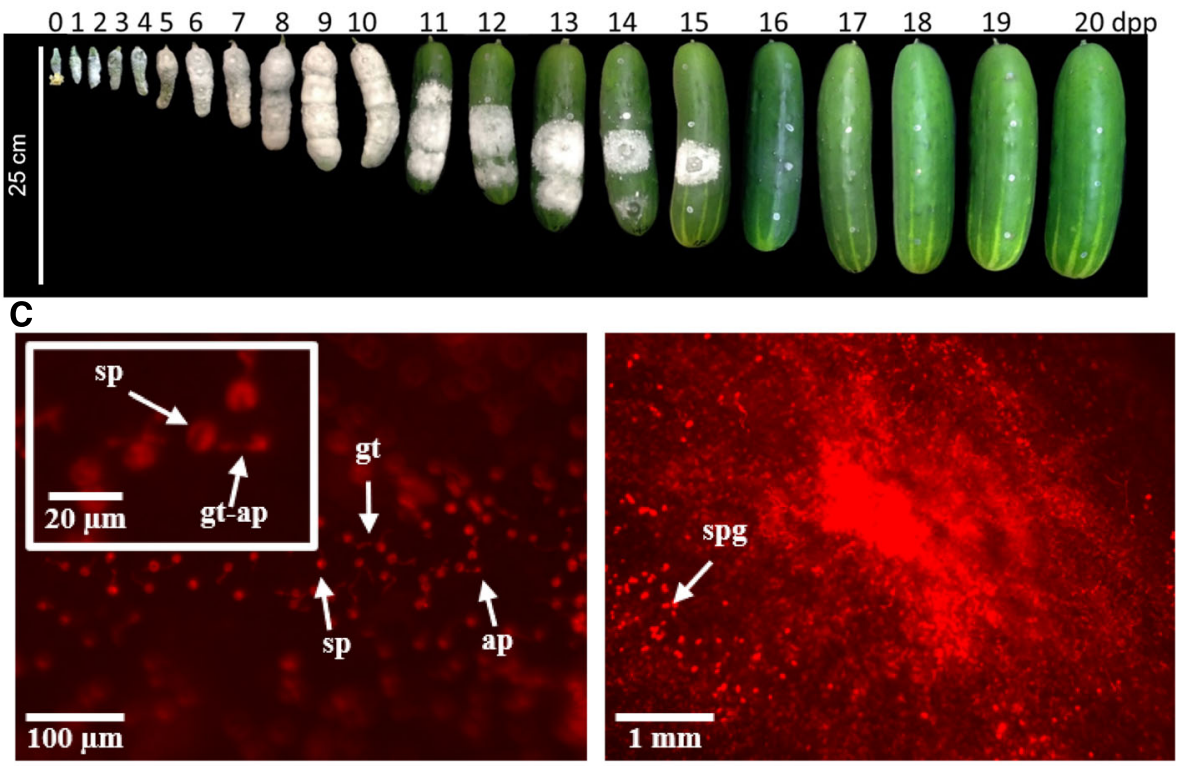

Fig. 1 Cucumber cultivar 'Poinsett 76 ' exhibits age-related resistance to $P$. capsici. a Fruit length and disease rating (DR) as a function of fruit age. Fruit were ranked on a 1-9 DR scale ( 1 = no symptom; $9=$ extensive mycelial growth and sporulation $)$ at 5 days post-inoculation. The dotted line at $D R=3$ represents localized necrosis. Points are means of 5-6 fruit. Error bars represent $+/-$ standard error of the mean. $\mathbf{b}$ Representative fruit and disease progression at 5 dpi. c Fluorescently labeled P. capsici on $<8$ dpp cucumber fruit at 4 (left) and 72 (right) hpi. sp. - spore; gt - germ tube; ap - appressoria; spg - sporangia

results (due to treatment of outlier genes in DESeq2), therefore analysis of differential gene expression was performed including the two samples.

\section{Resistant-age fruit mount a successful response by $24 \mathrm{~h}$ post inoculation}

To assess the changes in gene expression during infection we analyzed the transcriptional changes that occurred between consecutive timepoints at each fruit age ( 4 vs. 0 hpi, 24 vs. 4 hpi, and 48 vs. 24 hpi). Differential expression analysis showed that approximately 1800 genes were differentially expressed (up or down) at $4 \mathrm{hpi}$ compared to uninoculated tissue, for both the 8dpp and 16dpp fruit, indicating a rapid and strong response to infection at both ages (Fig. 2 b). However, as suggested by the PCA, progressive changes in gene expression were markedly different in the susceptible and resistant tissues at subsequent contrasts. While there was an increase in differentially expressed genes (DEG) in the susceptible $8 \mathrm{dpp}$ fruit peels with time, (4758 and 2505 


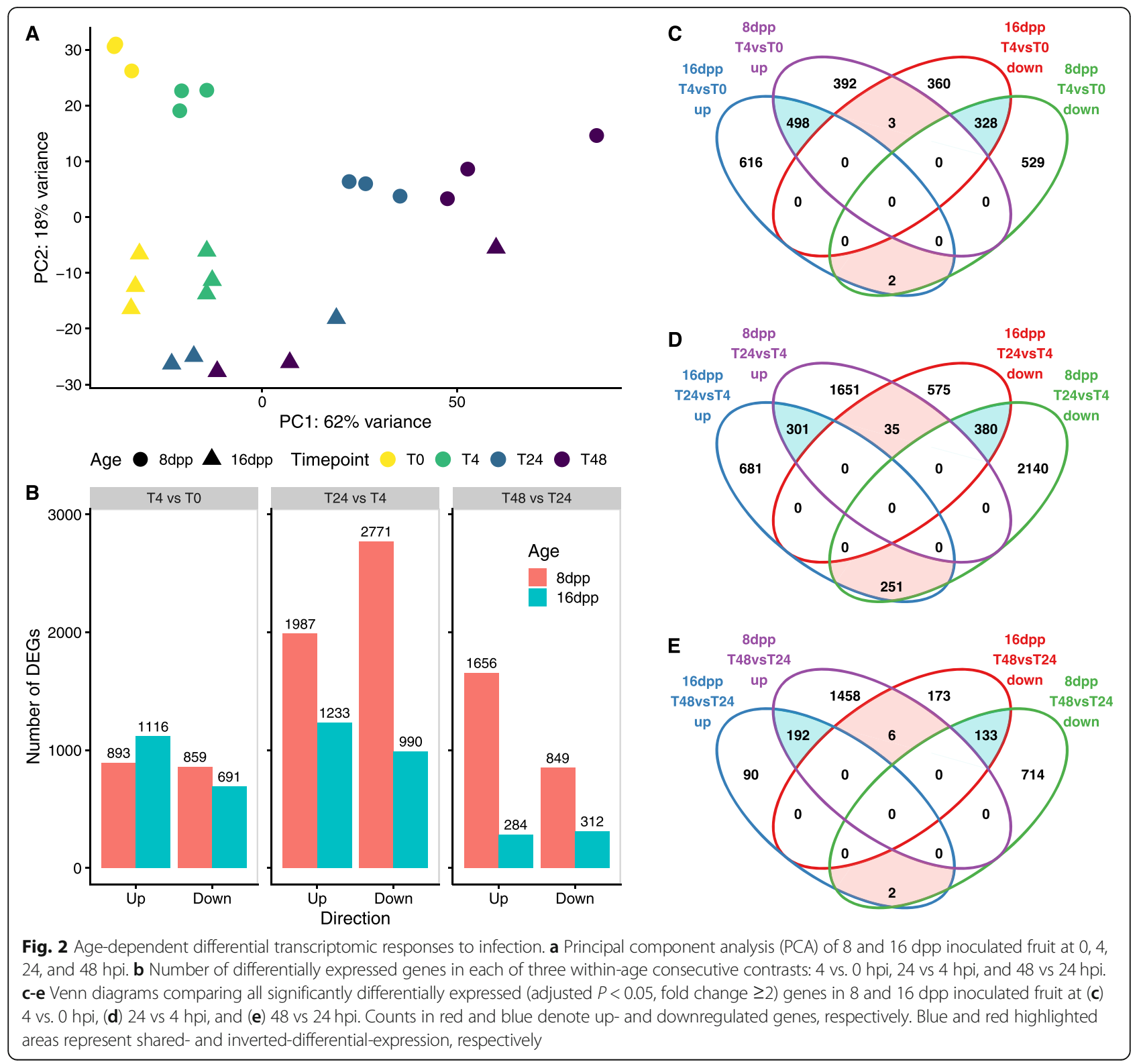

DEG at 24 and $48 \mathrm{hpi}$, respectively) the resistant $16 \mathrm{dpp}$ samples had a smaller number of DEG at $24 \mathrm{hpi}$ vs $4 \mathrm{hpi}$ (2223) and by 48 hpi only about 500 genes were differentially expressed compared to $24 \mathrm{hpi}$. This approach allowed us to identify key points of transcriptional change during early infection.

To further understand the biological processes involved in the two responses, the most significantly enriched GO-terms (Fisher-weight01 $p$-value <0.01) were compared for each of the consecutive contrasts (Fig. 3). At 4 hpi, upregulated genes in inoculated 8 and $16 \mathrm{dpp}$ fruit were strongly enriched for defense related genes. "Response to wounding", "defense response", "response to chitin", "phenypropanoid biosynthetic process, "response to oxidative stress", "response to karrikin", "organonitrogen compound catabolic process", "defense response to fungus", "aromatic compound catabolic process", and "drug catabolic process" were the ten most enriched terms in both ages at this time point. Although the number and GO categories of genes differentially expressed at 4 hpi was comparable between the two ages, fewer than half of the DEG in the resistant samples was shared with those differentially expressed in the susceptible samples (Fig. 2 c; blue shading). Analysis of the 613 genes uniquely upregulated in the resistant $16 \mathrm{dpp}$ samples at 4 hpi revealed a potentially unique set of defense related genes involved in an early incompatible interaction (Supplementary File 1).

When comparing $24 \mathrm{hpi}$ to $4 \mathrm{hpi}$, and $48 \mathrm{hpi}$ to $24 \mathrm{hpi}$, less than $15 \%$ of the thousands of up- and 


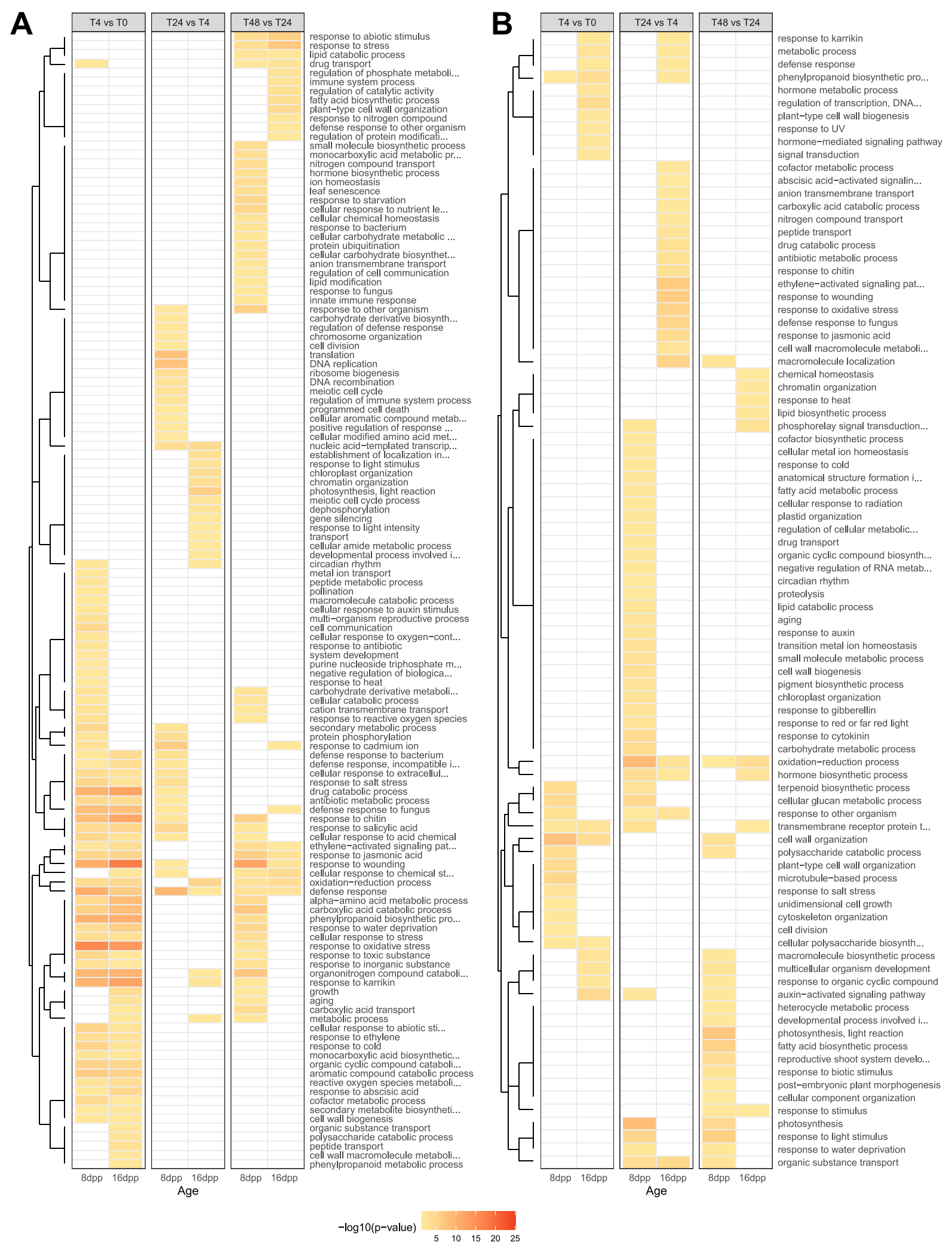

Fig. 3 Resistant-aged show transient upregulation of defense related genes. Gene Ontology enrichment of up- and down-regulated genes (a and $\mathbf{b}$, respectively). Each row represents an enriched term at one of the three consecutive contrasts: 4 vs. 0 hpi, 24 vs 4 hpi, and 48 vs 24 hpi. Enrichment pvalue threshold of 0.01 . Terms clustered by Euclidean distances 
downregulated genes were shared between the ages, respectively (Fig. $2 \mathrm{~d}$ and e). Susceptible $8 \mathrm{dpp}$ fruit continued to upregulate defense (top five enriched terms: "defense response", "DNA replication", "translation", "response to cadmium ion", and "response to salicylic acid") while down-regulating photosynthetic processes and other homeostatic processes, such as carbohydrate metabolic processes. In contrast, by $24 \mathrm{hpi}$, resistant $16 \mathrm{dpp}$ fruit were upregulating photosynthetic and growthrelated genes and downregulating defense (top five downregulated terms: "response to wounding" "ethyleneactivated signaling pathway", "macromolecule localization", "response to oxidative stress", "defense response to fungus") suggesting a return to normal state (Fig. 3 b). This is especially evidenced by the large number of inversely regulated genes in the $8 \mathrm{dpp}$ vs. $16 \mathrm{dpp}$ samples at $24 \mathrm{hpi}$ indicating an opposite response (red shading). The set of 251 genes upregulated in $16 \mathrm{dpp}$ and downregulated in $8 \mathrm{dpp}$ was strongly enriched for photosynthesis $(p$-value $=5.2 \mathrm{e}-13)$. Collectively these observations suggest that the resistant fruit have successfully mounted a defense by $24 \mathrm{hpi}$, indicating the importance of further investigation prior to that timepoint.

\section{Analysis of pathogen growth provides evidence for infection failure in the first $24 \mathrm{~h}$ on resistant fruit}

The transcriptomic suggestion of a rapid and potentially successful defense response within $24 \mathrm{~h}$ prompted us to more closely investigate pathogen growth during the first $24 \mathrm{~h}$ of infection using electron microscopy and a high throughput microplate assay. Samples were collected for Scanning Electron Microscopy (SEM) from 8 dpp and $16 \mathrm{dpp}$ aged cucumber fruit inoculated with droplets of zoospore suspension $\left(5 \times 10^{5}\right.$ spores $\left./ \mathrm{ml}\right)$ at time intervals of $0,2,4,8,12,18$, and 24 hpi. At each timepoint, three samples were collected, each from a distinct inoculated fruit. Morphological differences between $8 \mathrm{dpp}$ and $16 \mathrm{dpp}$ fruit were readily observed; the younger susceptible fruits had smaller more densely packed cells and trichomes, as well as warts that produced valley regions that in some cases increased spore density due to the surface topography (Fig. 4).

At 2 hpi, encysted P. capsici spores were observed germinating on fruit of both ages. Formation of some appressoria was also observed as early as 2 hpi. By 4 and 8 hpi, some differences were observable between the resistant (16 dpp) and susceptible (8 dpp) fruit. While spores on susceptible fruit continued to germinate and form appressoria, in four of the six $16 \mathrm{dpp}$ samples, lysed spores and germ tubes were observed, suggesting either preformed antimicrobial compounds or a rapidly induced defense response may inhibit successful infection as early as $4 \mathrm{hpi}$. As infection progressed, more evidence of failure to infect was observed in the majority of the resistant samples. By 18 and $24 \mathrm{hpi}$, deflated spores, germ tubes, and hyphae were observed on most of the resistant fruit samples, suggesting that spores that survived an initial defense response may be stopped at a later time, during the first $24 \mathrm{~h}$. No such histological signs of deflated or burst pathogen structures were observed at any timepoint in samples from susceptible 8 dpp fruit.

Fluorescence based in vivo infection assays provided further, quantitative evidence of inhibited infection in resistant aged fruit by $24 \mathrm{hpi}$. In these assays, fruit peel plugs (36 replicate fruit sections per fruit age, per experiment) were placed in a 96-well plate and inoculated with fluorescently labeled zoospores; fluorescence signal was measured hourly for $24 \mathrm{~h}$. After a short lag phase, the signal from the labeled $P$. capsici on inoculated susceptible $8 \mathrm{dpp}$ samples grew linearly throughout the 24-h period (Fig. 4 b). However, on $16 \mathrm{dpp}$ resistant-aged fruit samples, intensity of the fluorescent signal plateaued by 8-10 hpi, further suggesting early inhibition of pathogen growth. Together, the SEM and bioassay results bolster the transcriptional evidence suggesting that infection is thwarted by 24 hpi in $16 \mathrm{dpp}$ cucumber fruit.

\section{Transcriptomic investigation of the first $24 \mathrm{~h}$ post inoculation}

Concurrent with the collection of samples for $\operatorname{SEM}(0,2$, $4,8,12,18$ and $24 \mathrm{hpi}$ ), inoculated and uninoculated tissue was harvested for a second transcriptome analysis using 3' mRNA sequencing. In total, 78 3'-mRNA libraries were sequenced to an average depth of $\sim 5 \mathrm{M}$ reads/sample and an average of $\sim 53 \%$ reads quasi-mapping to the $3^{\prime}$-extended cucumber transcript sequences (Supplementary Figure 3). Two samples (8dpp_T12_Inoc_1 and 8dpp_ T18_Cont_2) had aberrantly low read coverage $(<0.5 \mathrm{M}$ reads) and were excluded from analysis. A PCA comparison of timepoints shared between the two transcriptome experiments $(0,4$ and $24 \mathrm{hpi})$ showed tight clustering of samples within their respective timepoints, indicating high reproducibility between the two experiments, performed in different years, and using different sequencing library technologies (Supplementary Figure 4).

The PCA of data from experiment 2 revealed modest changes in transcriptomic patterns for uninoculated samples of both ages (open symbols) relative to timepoint 0 (asterisks), likely reflecting a combination of diurnal changes and the effects of fruit detachment from the vine (Fig. 5). In contrast, the inoculated samples (closed symbols) showed strong transcriptional changes, especially for the susceptible $8 \mathrm{dpp}$ fruit (circles) (Fig. 5 a). From 4 hpi and beyond, the inoculated 8 dpp samples exhibited a sequential transcriptomic transition. Conversely, samples collected from the resistant-aged $16 \mathrm{dpp}$ 


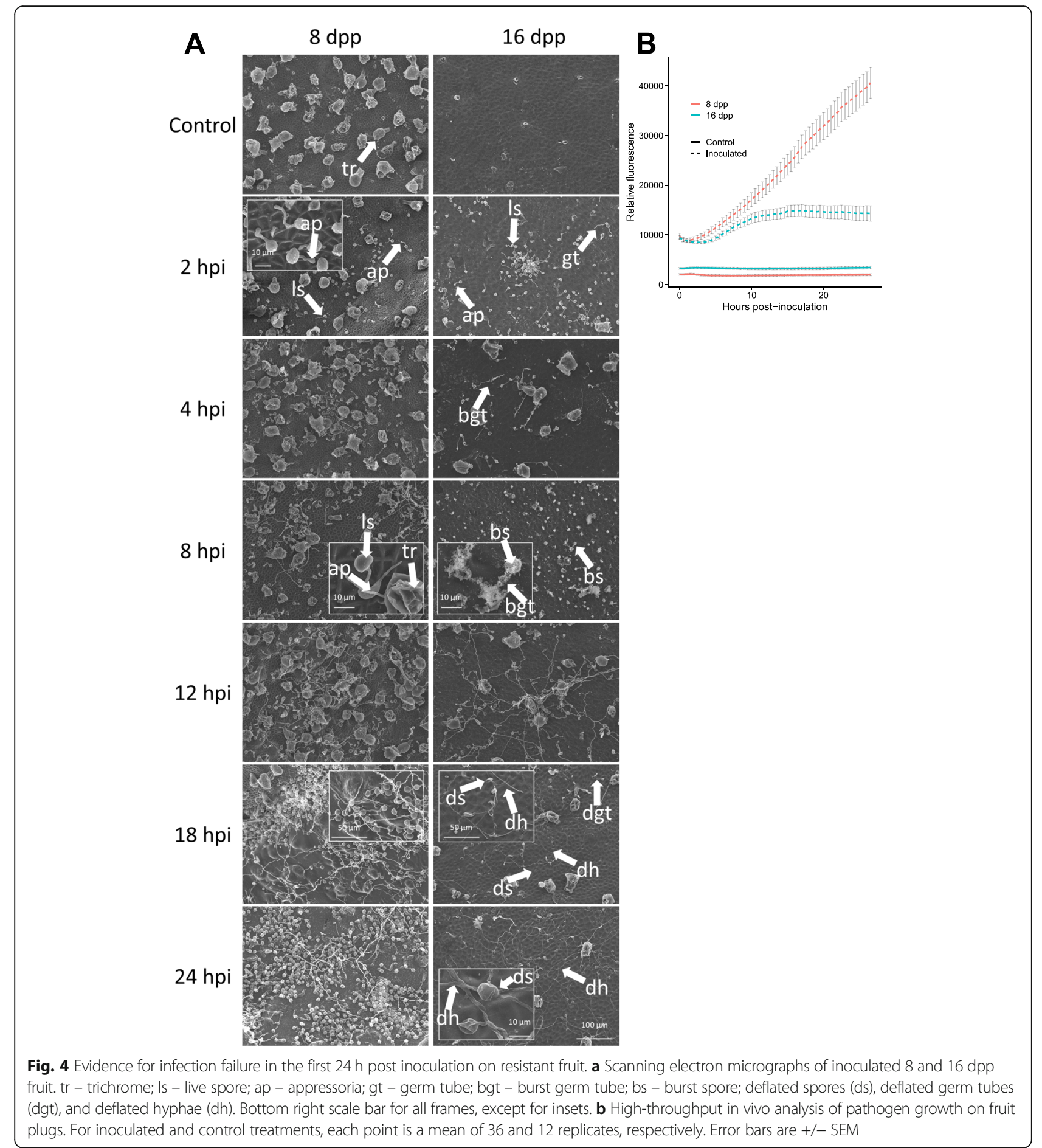

fruit (triangles) all clustered together, and relatively closely to uninoculated fruit, from 4 hpi and beyond. Notably, at 2 hpi, samples from uninoculated $8 \mathrm{dpp}$ fruit clustered with uninoculated control, while 2 hpi samples from $16 \mathrm{dpp}$ fruit showed a clear difference from the uninoculated controls, suggesting an earlier response to infection in the resistant-aged fruit.
To further understand the trends in gene expression changes in response to infection, all genes that were differentially expressed in at least one timepoint vs. the respective non-inoculated control were displayed as stacked bars (strata) in an alluvial plot (Fig. 5 b). The alluvia (curves) illustrate progressive changes in gene expression over time post-inoculation ( $2 \mathrm{~h}$ to $24 \mathrm{~h}$ ); each 


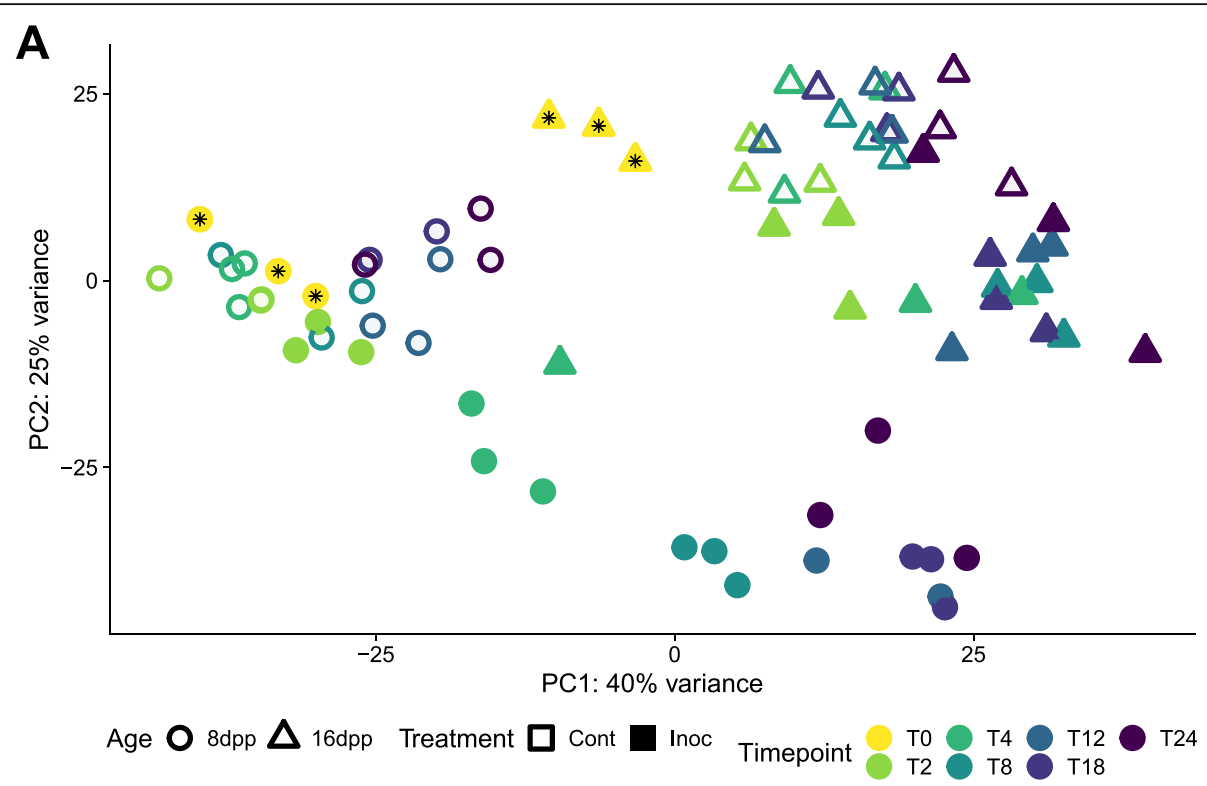

B

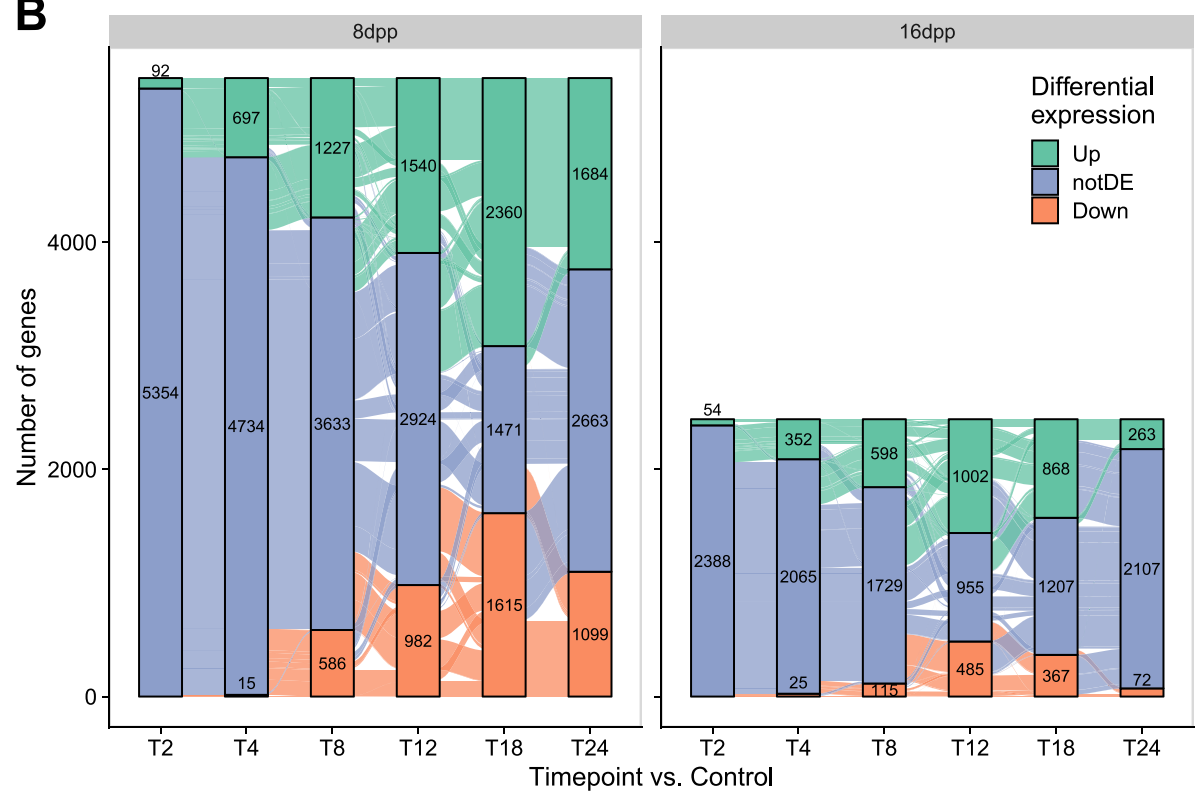

Fig. 5 Differential transcriptional dynamics within the first $24 \mathrm{~h}$. a Principal component analysis of inoculated and control fruit of two ages, 8 and 16 dpp. Samples collected at time 0, which represent both Inoculated and Control treatments, are denoted by an asterisk. b Alluvial plots showing all genes with significantly changed expression at each timepoint compared to respective controls. Each stacked bar represents the numbers of genes either up-down- or not-differentially (notDE) regulated at sequential time points. Genes are grouped based on expression patterns throughout time

alluvium represents a group of genes that shares expression pattern over time. The large alluvia from the nonDE strata (blue) show a phased response to infection, in which certain genes are involved in early timepoints, while others in later stages of infection.

The figure reveals that response to infection is dramatically different in the two ages. Most evident is that more than double the number of unique genes is differentially expressed at least once in susceptible fruit compared to resistant fruit. Examination of the trends in 8 dpp susceptible fruit revealed a pattern of sequential accumulation of DEG, as previously suggested by the PCA. The number of DEG grew continuously until $18 \mathrm{hpi}$, and most genes which were differentially expressed at one timepoint continued to be differentially expressed at following timepoints. Of the 697 upregulated genes at 4 hpi, 424 are continuously upregulated in every subsequent timepoint. By $24 \mathrm{hpi}, 1523$ of the 1684 upregulated genes had been previously upregulated at one of the timepoints and then subsequently upregulated at all 
following timepoints. A similar trend is observable in downregulated genes.

Conversely, by following alluvia of genes initially upregulated at 4 hpi in the $16 \mathrm{dpp}$ resistant-aged samples, it is evident that most are subsequently not differentially expressed at further timepoints. Of the 352 genes upregulated at $4 \mathrm{hpi}, 140$ genes are not differentially expressed at $8 \mathrm{hpi}$, and only 45 are continuously upregulated through 24 hpi. Though the number of DEG grows until $12 \mathrm{hpi}$, most genes are timepoint specific, being differentially expressed once or at most at two timepoints. Most striking is the fact that at $24 \mathrm{hpi}$, only 263 and 72 genes are up- and down-regulated, respectively, compared to uninoculated tissue, further confirming the culmination of the defense response in resistant-aged fruit.

\section{Gene co-expression is preserved but not expression patterns over time}

To better identify transcriptional co-expression patterns in the data we employed Weighted Gene Co-expression Network Analysis (WGCNA) [26]. This analysis identifies genes with highly correlated transcriptional patterns and groups them into co-expression clusters or modules. These modules help understand biological processes and identify key genes associated with these processes [26]. Two independent response networks for the susceptible and resistant fruit (Fig. 6) were assembled. After module merging (based on eigengene correlation), a total of 17 and 29 modules were defined for the susceptible and resistant inoculated networks, respectively (Supplementary Files 2 and 3).

Module preservation statistics [27] showed that gene co-expression was preserved in many modules when comparing the susceptible and resistant networks (Supplementary Figure 5). Module gene overlap (Fig. 6 c) also showed significant preservation (Fisher's Exact test and FDR $<0.001$ ) of gene co-expression between many modules of the two networks. For example, close to half of the expressed genes in the susceptible response are clustered in the Susceptible Module 1 (S1) which is consistently downregulated until 18 hpi (Fig. 6 d). The genes in this module overlap with subsets of genes in six resistance modules (R2, R3, R6, R8, R12 and R17), indicating they are also co-expressed in the resistant network, but in a different manner. For instance, a subset of the co-expressed genes present in module $\mathrm{S} 1$ are coexpressed in resistant-age fruit in module R12; however, while the genes in S1 show a drop in expression, in resistant fruit they have minimal fluctuations in expression over time. Thus, while many groups of genes were expressed in concert over time, indicating coordinate regulation regardless of fruit age, the diverse module assignment and thus age-specific patterns of expression, suggests a reprogramming of the response network in resistant-aged fruit.

\section{Biological processes identified by weighted gene co- expression network analysis}

Using gene module assignment defined by the resistant network, expression patterns of the genes within a given module were compared in control and inoculated fruit of both ages (Fig. 7). Genes identified in many of the coexpression modules of infected fruit had distinct patterns from those in uninoculated control fruit, suggesting we were able to isolate specific defense responses in response to inoculation.

GO-term enrichment analysis of the different modules demonstrated the biological relevance and function of identified modules (Supplementary File 4). For example, Module R1 (Resistance Module 1) (n genes = 3013) showed patterns of increased expression in response to inoculation in both ages. Uninoculated tissue largely showed unchanging expression levels throughout the entire time course. GO term enrichment showed that this module was strongly associated with translation and ribosome biogenesis, suggesting induction of protein synthesis in response to infection.

In Modules R2 and R9 (n genes $=1598$ and 625, respectively) all genes, regardless of age or treatment, showed a similar pattern of change over time. Genes in Module R2, which exhibited gradual decline, were enriched for carbohydrate metabolic process, lipid metabolic process, cell division, signal transduction and response to abiotic stimulus. Higher baseline expression of $8 \mathrm{dpp}$ fruit is likely due to the different stage of development, as unharvested fruit at this age are still rapidly growing (Fig. 1 b). Genes in Module R9 showed gradual increase and were enriched for "organonitrogen compound catabolic process", "aromatic compound catabolic process" as well as" response to water deprivation". The expression patterns of genes in these modules is probably a result of the fruit being detached during the analysis, i.e. deprived of carbohydrate source and subject to water loss.

Module R3 (n genes $=1575$ ) showed a potentially circadian controlled expression pattern, with peak expression at $18 \mathrm{hpi}$ (collected at 6:30 AM; sunrise at 6:15 AM) and almost identical patterns of expression in uninoculated fruit of both ages. In inoculated fruit of both ages, the circadian patterns of these genes were diminished, and they showed a prompt drop in expression levels after inoculation. By 24 hpi however, expression levels in resistant fruit returned to levels of uninoculated fruit, while those in susceptible fruit remained low. This module was enriched for processes involved in transcriptional regulation as well as response to light stimulus. 


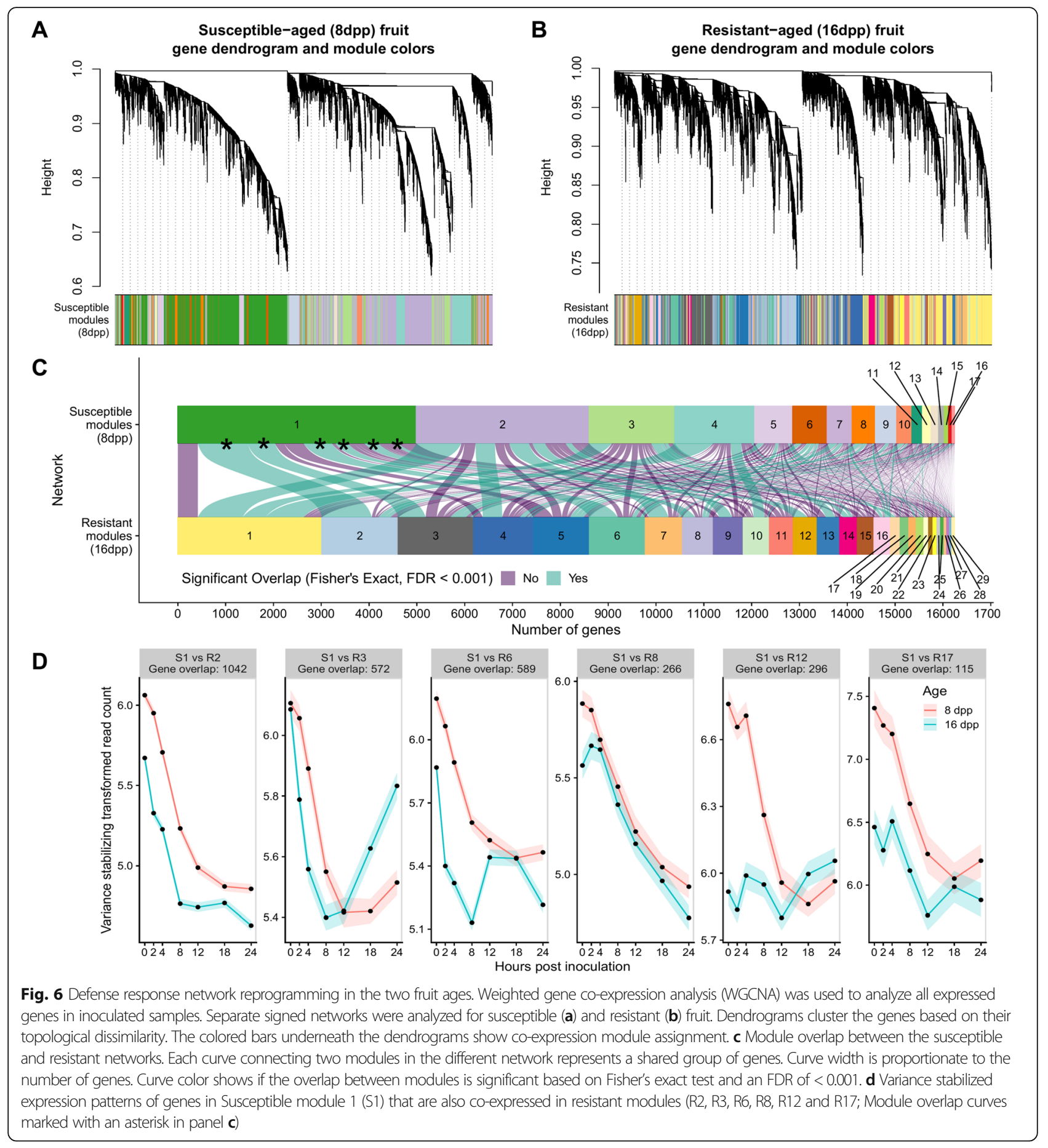

Modules induced in early infection of resistant-aged fruit Several modules indicated differential response to infection between the resistant and susceptible ages. Based on the PCA and microscopy, we were especially interested in modules that showed very early response differences. To identify such modules, expression patterns of genes, in the resistant network modules, were compared between $8 \mathrm{dpp}$ and $16 \mathrm{dpp}$ samples using regression analysis. Because gene expression patterns do not follow linear trends over time, a cubic spline basis function was applied to the time variable with three internal knots, splitting the time course into quartiles $(0-3,3-8,8-15$, and 15-24 hpi). Most of the modules (24/29) showed significant interaction effects between age and splined modeled time in at least one quartile, indicating that genes in each module behave differently across time in 


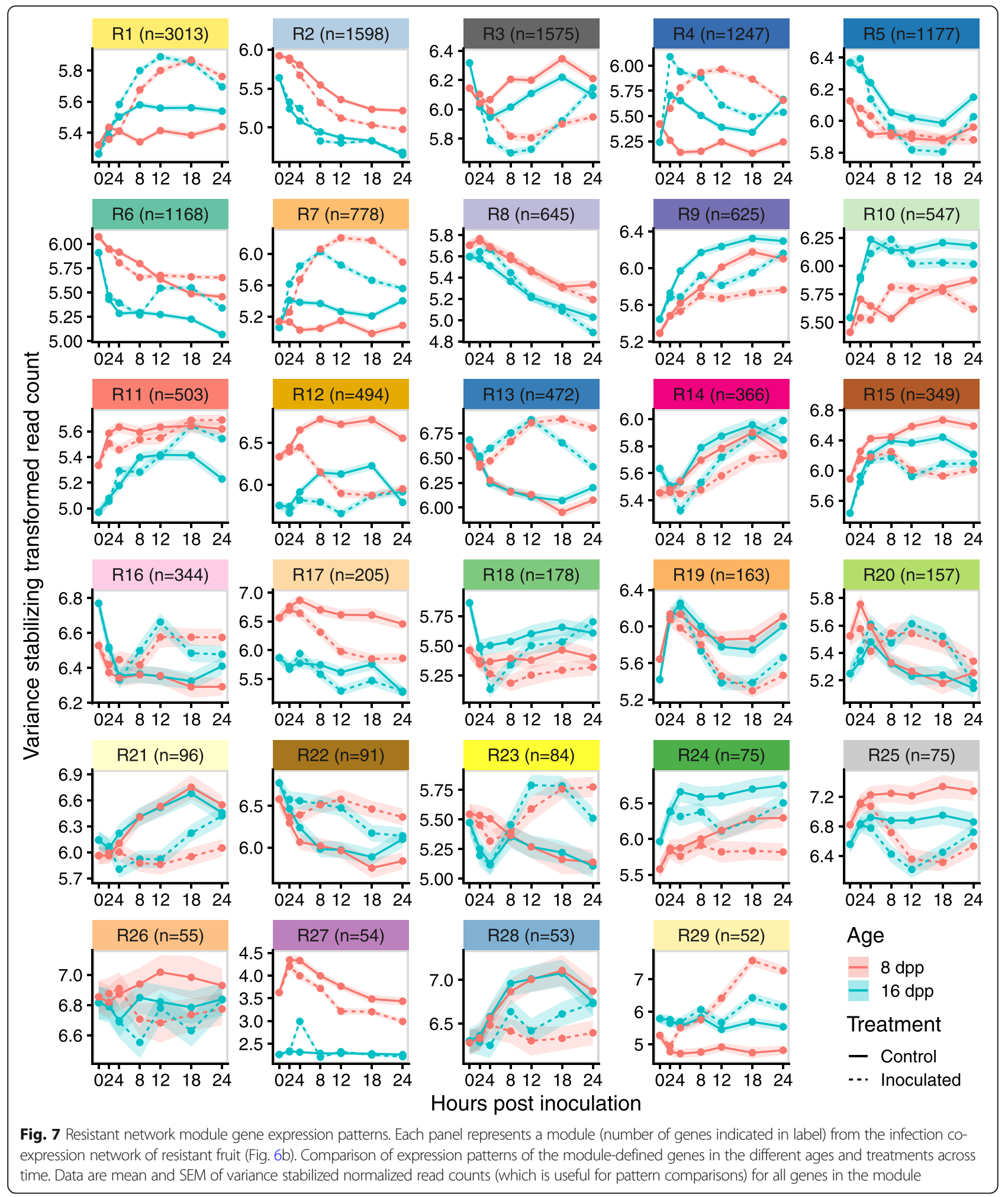

the two ages, further reflecting the reprogramming of module expression patterns (Supplementary Table 3). Of specific interest were early induced modules in $16 \mathrm{dpp}$ peels vs. 8 dpp peels. Nine modules had significant interaction effects during the first two spline fractions, 0-3 hpi and 3-8 hpi (Fig. 8).

Module R4 $(n=1247)$, is defined by a spike of increased expression in response to inoculation of 


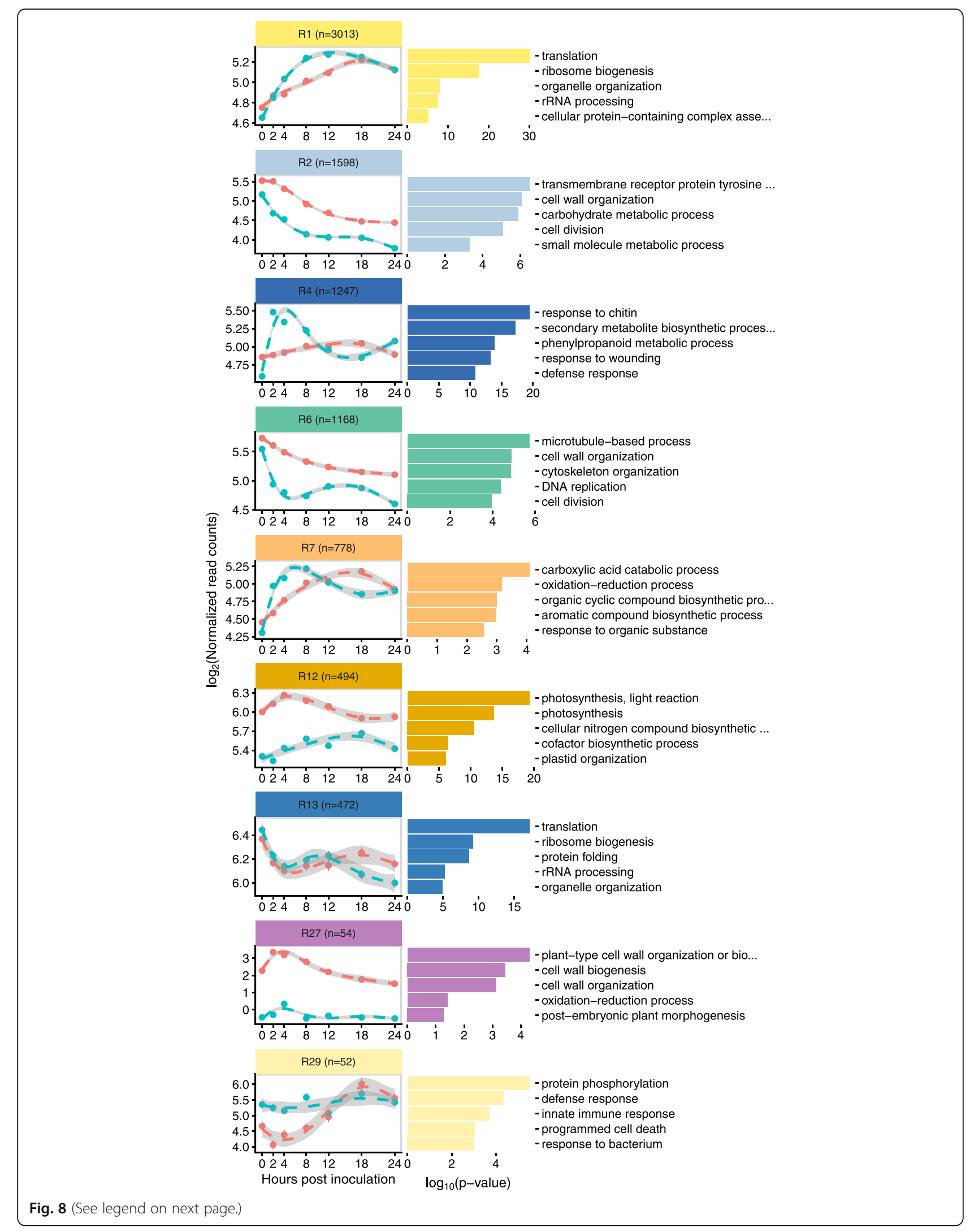


(See figure on previous page.)

Fig. 8 early response modules in the resistant network. Points represent the mean $\log _{2}$ (normalized read count) of inoculated $8 \mathrm{dpp}$ or $16 \mathrm{dpp}$ fruit. Lines and grey ribbons are predicted values (and standard errors) based on regression of $\log _{2}$ (normalized read count) by a natural cubic spline of time with three internal knots $(0-3,3-8,8-15$, and 15-24 hpi). Modules selected are those with a significant interaction effect in one or both first spline fractions ( $0-3$ and 3-8 hpi), i.e. have age-dependent response to infection in early timepoints. The five most significantly enriched gene ontology terms are indicated next to each module

resistant-aged fruit. Expression levels peak at 2 and 4 hpi followed by decrease in expression starting at $8 \mathrm{hpi}$. In the inoculated susceptible fruit, the genes identified in this module showed a minimal change in expression prior to 4 hpi. GO term enrichment showed that this module was strongly associated with defense and pathogen recognition, the five most enriched terms being "response to chitin", "secondary metabolite biosynthetic process", "phenylpropanoid metabolic process", "response to wounding", "defense response".

Similarly, Module R7 $(n=778)$ also showed statistically different expression during early infection, with increased expression by 2 and 4 hpi in inoculated resistant-aged fruit. In susceptible fruit these genes show a more gradual increase in expression, which only matches that of the resistant-aged fruit at $8 \mathrm{hpi}$. This module was also strongly associated with defense as evidenced by enrichment for terms associated with canonical response mechanisms "carboxylic acid catabolic process", "oxidation-reduction process", "organic cyclic compound biosynthetic process", "aromatic compound biosynthetic process".

Other interaction effects and expression patterns of note are observed in other modules. Module R1, which as mentioned earlier is strongly enriched for translationrelated processes and is activated earlier and more rapidly in inoculated resistant-aged fruit. A more rapid reduction in expression in genes of Module R6 (enriched for microtubule, cell wall and cell division related processes) is observed in resistant-aged fruit, suggesting a rapid response. Genes in Module R29 which are associated with defense and innate immune responses, are already activated at $0 \mathrm{hpi}$ and show little change in expression in resistant-aged fruit. Interaction effects in Modules R2, R12 and R27 which are enriched for growth, cell wall and photosynthesis related terms, are most likely associated with baseline differences between the different ages.

\section{Identification of early response genes in resistant-aged fruit}

Genes with high module membership are strongly correlated to the module eigengene. We used this measure to identify genes highly connected to Modules R4 and R7, as these modules showed patterns of increased expression at early infection timepoints in resistant fruit. The second criterion for selection was those genes that also showed significantly increased expression in resistant inoculated fruit at 2 and/or 4 hpi compared to both the uninoculated control and the inoculated susceptible fruit. We identified 34 genes with a greater than 2 -fold expression at 2 and/or $4 \mathrm{hpi}$ in both comparisons as well as a module membership greater than 0.75 (Table 1 , Supplementary Figure 6). As expected, the expression patterns of these genes strongly conform to those of the Module eigengene, with a uniquely strong expression at early timepoints in resistant inoculated fruit. Many of these genes are annotated to have canonical functions in early defense response, for example associated with phenylpropanoid-derived compound metabolism, reactive oxygen species (ROS) metabolism, gibberellin and ethylene balance, vesicle transport, protein phosphorylation, as well as pathogen perception and response (Fig. 9). The expression patterns observed for these genes (i.e., earlier increase in 16 dpp samples, but higher level at $24 \mathrm{hpi}$ in $8 \mathrm{dpp}$ samples) were confirmed by comparison to their expression in RNA-seq experiment 1 (Supplementary Figures 7 and 8). These data showed strong reproducibility (Pearson's correlation; $R=0.85, p$ value $<2.2 \mathrm{e}-16)$ in response between experiments performed in different years and with different sequencing library techniques.

\section{Discussion}

\section{A rapid infection meets a rapid response}

The infection process of Phytophthora spp. has been studied extensively on many plants and crops [28]. However, there are clear differences between the rates and severity of infection in different species and hosts. For example, $P$. infestans infections on potato and tomato are largely symptomless until 3 days post infection (dpi) [29]. As such, many transcriptome studies examine much later time points post inoculation [e.g., [30, 31]]. In contrast, $P$. capsici has a rapid infection cycle with visible symptoms, often within $24 \mathrm{hpi}$, and can reach asexual sporulation by $2-3$ dpi on a variety of species $[6,18]$.

Using both fluorescent microscopy and SEM, we observed that $P$. capsici infection of susceptible young cucumber fruit is also extremely rapid. On susceptibleaged fruit, hyphal growth was observed as early as $8 \mathrm{hpi}$ and progressed rapidly throughout the first $24 \mathrm{~h}$, indicating a successful biotrophic infection. Using in vivo highthroughput bioassay, we quantitatively observed a detectable linear growth rate on cucumber fruit peel 


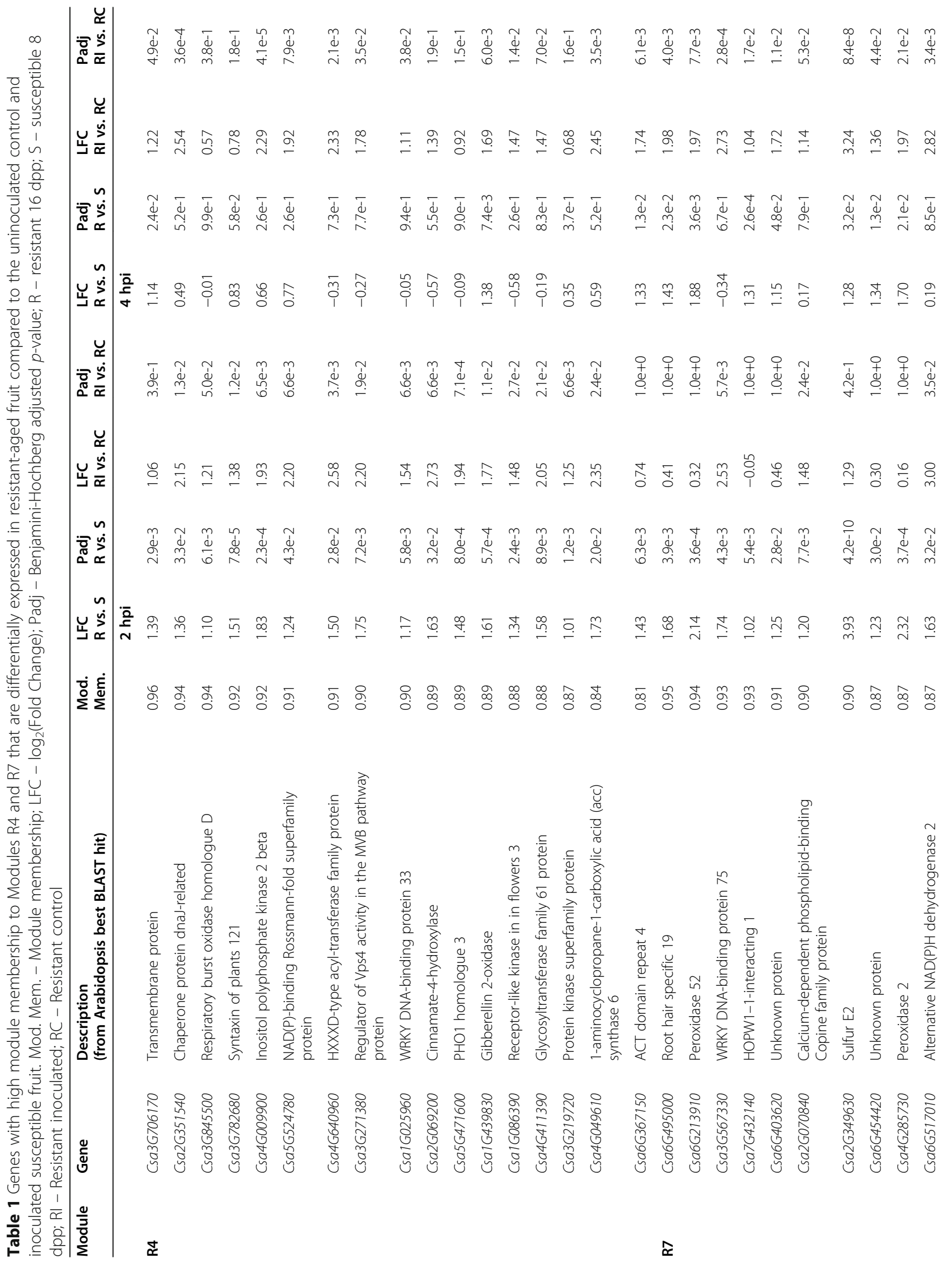




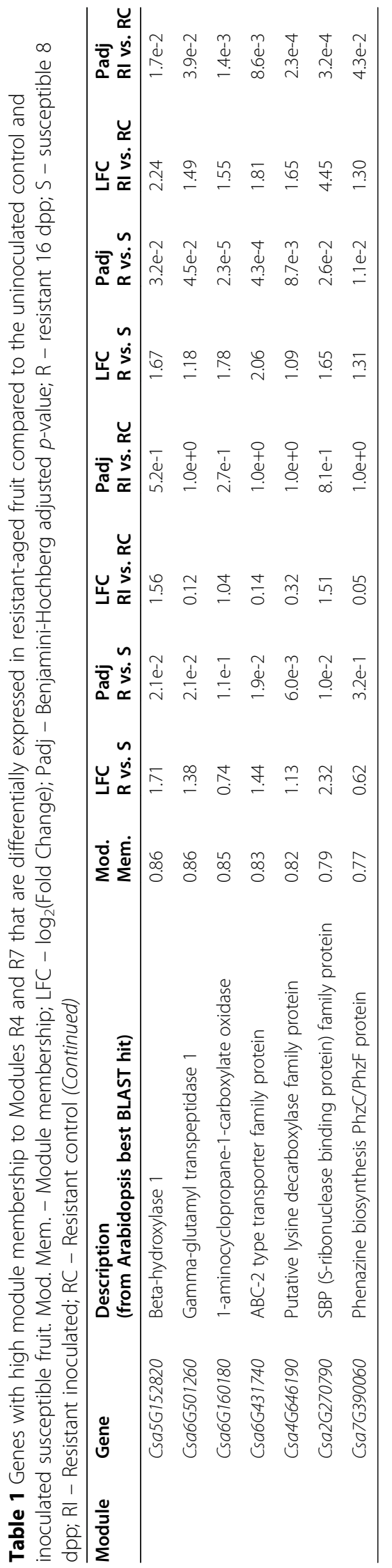




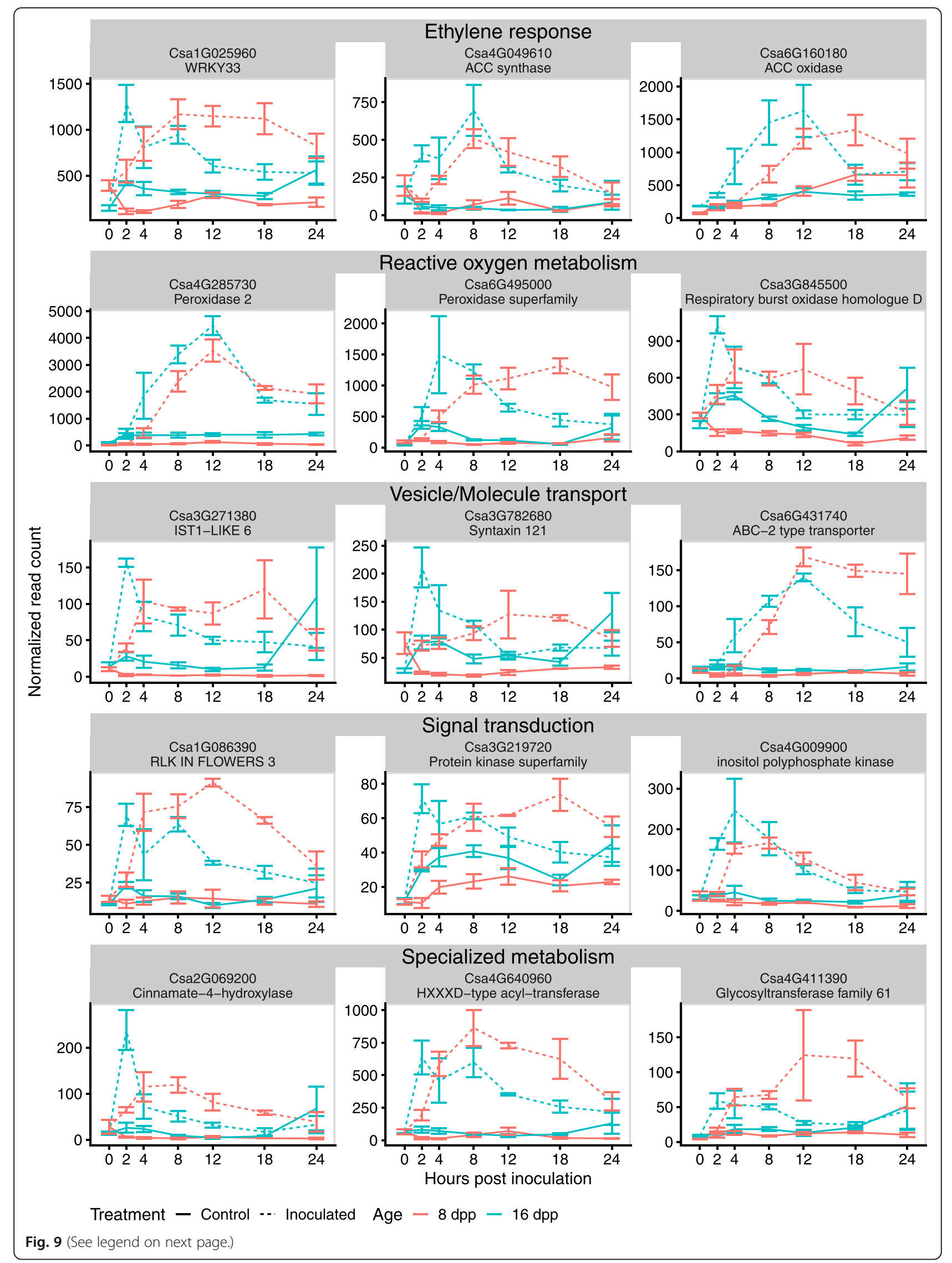


(See figure on previous page.)

Fig. 9 Genes induced in early responses to inoculation of resistant fruit. A subset of genes identified by a module membership $>0.75$ and significant fold change of $>2$ in inoculated resistant fruit ( $16 \mathrm{dpp}$ ) compared to both control resistant fruit and inoculated susceptible fruit ( $8 \mathrm{dpp})$. Full list and expression patterns of identified genes available in Table 1 and Supplementary Fig. 6 . Mean expression for three biological replicates in each age and treatment. Error bars are $+/$ - standard error of the mean

samples as early as 4-6 hpi, again suggesting quick establishment and rapid progression during this time. As described on other hosts [19], our fluorescent microscopy showed that by $72 \mathrm{hpi}$ asexual reproductive sporangia have been formed in cucumber. It is this rapid infection and reproductive cycle that allows $P$. capsici to be such a devastating pathogen in the field.

Our prior studies have shown that certain cucumber cultivars and plant introgression lines [13-15] exhibit ARR at approximately $16 \mathrm{dpp}$, coinciding with the end of exponential fruit growth. Varieties that do not develop ARR remain highly susceptible to $P$. capsici and show severe symptoms of infection [13-15]. Evidently, inhibition of zoospore germination is not the mechanism of ARR; regardless of fruit age, within $2 \mathrm{~h}$ of inoculation most $P$. capsici zoospores had already encysted and germinated (Fig. 4), and some had formed appressoria. Strikingly however, the SEM of inoculated resistant fruit revealed histological signs, such as burst or lysed spores and germ tubes, as early as $4 \mathrm{hpi}$, and consistently by 8 hpi. Further confirmation of rapid inhibition of infection on the $16 \mathrm{dpp}$ fruit was obtained from our quantitative in vivo bioassay showing cessation of pathogen growth by $8-10 \mathrm{hpi}$.

By $24 \mathrm{hpi}$, all three resistant-aged samples examined with SEM either showed no pathogen present, suggesting failure to remain attached to the fruit surface or deflated/unviable spores and hyphae. None of these histological signs were present in susceptible samples, on which infection proceeded normally. Similarly, a study of Port-Orford-cedar plants resistant to $P$. lateralis showed a reduction in pathogen presence as well as deflated hyphae and spores at $24 \mathrm{hpi}$ [32]. Though there is limited histopathological evidence of such a severe defense response, similar signs of Phytophthora spp. (including capsici) inoculum death are observed after exogenous application of phytochemicals such as garlic root exudates or terpenoid-containing essential oils from oregano and other plants [33-36]. Evidence in other fungal pathosystems of spore- and hyphal membrane disruption by preformed or induced defensin proteins also exists [e.g., [37, 38]]. This study and our prior work showed an upregulation of specialized metabolism associated genes in 16 dpp vs. 8 dpp uninoculated fruit peels. Thus, the rapid histological signs of pathogen death might result from preformed antimicrobial compounds, as was similarly implicated from peel extract assays and transcriptomic analysis of non-inoculated developing peels of other ARR cultivars [14, 15], or from a rapidly induced defense response.

The findings indicating rapid inhibition of infection were further supported by transcriptional evidence. Gene expression changes over time were markedly different between resistant and susceptible aged fruit; double the number of genes were involved in the susceptible response compared to the resistant one. Moreover, susceptible fruit exhibited progressive waves of gene expression changes peaking at $18 \mathrm{hpi}$, many associated with defense response (Figs. 3 and 6 a). The susceptible response may be compared to a high-resolution transcriptional time series of the compatible response of Arabidopsis to infection by the fungal pathogen Botrytis cinerea [39]. In Botrytis infection of Arabidopsis, most differential expression also occurred at $\sim 18-30 \mathrm{hpi}$. As these timepoints are post-pathogen penetration, the increased gene expression at these times may represent a response which is too late to inhibit infection [39]. The increased expression at comparable timepoints in our data might suggest a similarly failed defense response in susceptible-aged fruit. Additionally, as was seen in successful infection of tomato by $P$. capsici [19], the susceptible cucumber fruit also exhibited down-regulation of genes associated with primary metabolism processes and photosynthesis in by $24 \mathrm{hpi}$.

Conversely, in resistant-aged fruit, an induced rapid activation of defense related genes at 2 and 4 hpi was followed by active downregulation of defense related genes by $24 \mathrm{hpi}$. Furthermore, at $24 \mathrm{hpi}$, relatively few genes were differentially expressed in inoculated resistant fruit compared to the control (Fig. 5 b), providing an additional indication that the pathogen defense response is largely completed. This downregulation of defense makes intuitive sense if the pathogen has been eradicated or is no longer infecting the host, as was observed in the microscopic and in vivo assays. Apart from downregulation of defense, resistant-aged fruit showed upregulation of photosynthesis and other metabolic processes, suggesting a return to a "normal" or uninfected state after pathogen defeat. Rapid responses to $P$. capsici have also been observed in incompatible reaction on Arabidopsis leaves where failure to penetrate, ROS bursts, callose deposition, and hypersensitive cell death all occurred within $24 \mathrm{hpi}$ [40]. Together the evidence from microscopy, bioassay and transcriptome studies 
suggested that factors, either pre-formed or induced, prior to $24 \mathrm{hpi}$ are important in conferring ARR.

\section{A reprogramming of gene co-expression networks of infection at the resistant age}

To better understand the effect of fruit age on gene expression patterns in response to $P$. capsici inoculation, the high-resolution transcriptomic time course was further analyzed using WGCNA [26]. This advanced analytical approach, which identifies gene co-expression modules, together with the unique ability afforded by an ARR pathosystem to examine both susceptible and resistant responses within the same plant genotype, helps us gain valuable insight into the mechanisms that confer plant disease resistance.

When comparing the gene co-expression networks of the resistant and susceptible interactions, we first observed a large difference in the number of modules identified in each network. Furthermore, module preservation patterns suggested that the co-expression response in resistant-age fruit was reprogrammed as compared to the network on susceptible fruit (Fig. 6). While co-expressed sets of genes share similar regulatory mechanisms, these are employed at different times and with different patterns during the infection of resistant and susceptible-aged fruit. Network reprograming in responses to infection by $P$. syringae was similarly observed when comparing resistant wild type plants and effector-triggered-immunity compromised mutants of Arabidopsis [41]. Specifically, similar groups of genes were shown to be activated in compatible- and incompatible-interactions; however, their timing and expression patterns were altered.

While we identified several modules that exhibit similar expression patterns regardless of infection (i.e. likely a response of fruit detached from the plant), most modules were impacted by infection (Fig. 7). The differences in expression patterns observed between inoculated and non-inoculated tissue reveal we were successful at identification of defense response co-expression modules. We focused our analysis on the differences in gene expression patterns of genes identified to be induced earlier by inoculation in the resistant network. In PCA, susceptible samples at 2 hpi clustered with noninoculated controls (Fig. 5 a). Conversely, resistant-aged samples at 2 hpi showed a distinct transition along both PC1 and 2, away from the uninoculated controls, suggesting a more rapid transcriptional defense response in these samples. We thus were interested in modules showing differential expression patterns at very early timepoints. As optimal timing of defense response can be crucial for successful resistance [42], the ability to mount a successful defense could be attributed to this early response.
By performing cubic spline regression on module gene expression curves, we identified gene co-expression modules associated with defense that are differentially activated in resistant-aged fruit as early as 2 and 4 hpi (Fig. 8). Of specific interest were module R4 and R7; in both cases increases in gene expression were delayed in susceptible relative to resistant-aged fruit. Genes of interest were identified using a combination of module membership statistics and differential expression analysis (Fig. 9). Among the 34 candidate genes of interest showing resistance-specific increase at 2 or $4 \mathrm{hpi}$, were two WRKY transcription factor homologs Csa1G025960 (AtWRKY33, BLAST E=2.8e-119) and Csa3G567330 (AtWRKY75, BLAST E=7.4e-44). While AtWRKY75 is reported to be associated with phosphate deficiency [43], its cucumber homolog may function in defense to pathogen infection. AtWRKY33, however, has been shown to be important in resistance to Alternaria brassicicola and Botrytis cinerea in Arabidopsis [44, 45]. AtWRKY33 is also rapidly induced by the flg22 epitope as part of microbe-associated molecular pattern immunity, with downstream targets involved in ethylene and camalexin synthesis as well as other transcription factors and pathogen receptors [46]. Furthermore, in a proteomic study of $P$. capsici infection, the tomato WRKY33 homolog protein was found to be induced by $8 \mathrm{hpi}$ and localized to the nucleus [47].

Among the targets of WRKY33 in Arabidopsis are ethylene biosynthesis genes [46]. We further identified that the two ethylene synthesis genes Csa4G049610 and Csa6G160180, encoding 1-aminocyclopropane-1-carboxylate synthase and 1-aminocyclopropane-1-carboxylate oxidase, respectively, both have high module membership with Modules R4 and R7 respectively. Although ethylene is generally thought to be important in defense response against fungi and necrotrophic pathogens [48], ethylene response, but not SA or JA, was shown to be crucial for inhibition of $P$. capsici growth in habanero pepper, [49]. Blocking ethylene perception by means of exogenous application of silver nitrate reduced this inhibition [49]. Moreover, silencing of ethylene signal transduction in Nicotiana benthamiana resulted in loss of ARR to P. infestans [50]. The uniquely increased expression of the cucumber WRKY33 and downstream upregulation of ethylene synthesis observed in resistant fruit could thus be a central component in regulation of the successful defense response in cucumber ARR.

Consistent with a successful hypersensitive response in resistant-age fruit, this group of early induced genes also included three putative peroxidases (Csa6G213910, Csa4G285730 and Csa6G495000), an NAD(P)H-dehydrogenase (Csa6G517010), as well as an NADPH/respiratory burst oxidase protein (Csa3G845500) that could all potentially serve in modulating ROS within the first few hpi 
[51]. Other genes identified to be potentially involved in defense are genes involved in vesicle transport (Csa3G271380, Csa3G782680 and Csa6G431740), as well as a signal transduction (Csa1G086390, Csa3G219720 and Csa4G009900) and specialized metabolism (Csa2G069200, Csa4G640960 and Csa4G411390). Upregulation of Csa2G069200, a putative cinnamate-4-hydroxylase, could be acting either in specialized metabolite synthesis, or perhaps upstream of other enzymes in a lignification response. Finally, Csa2G070840, which putatively encodes a calcium-dependent phospholipid-binding copine family protein, might also be important in fine-tuning the response to infection, as its homolog in Arabidopsis functions in stomatal closing during infection and regulation of several resistance receptor genes [52, 53]. All these genes are canonically involved with response to pathogens, and so their early activation in response to inoculation in resistant fruit could be crucial in conferring ARR by limiting pathogen establishment in early stages of infection.

\section{Cucumber ARR to P. capsici may be mediated by developmental regulation of basal defenses and receptor- like genes}

While it is tempting to speculate that resistances in young cucumber fruit may have been lost as a trade off in breeding for rapid growth, screening of the highly diverse USDA cucumber germplasm collection suggests against this idea, as the great majority are highly susceptible when very young [20]. On the other hand, while not all lines show as dramatic an ARR to $P$. capsici as 'Poinsett 76' or 'Vlaspik', other cultivars and wild accessions do show increased expression of defense genes after exponential growth [15], as well as a tendency to become less susceptible as they approach full size [13]. This further suggests that ontogenic defenses in cucumber fruit may result from a combination of an increase in preformed basal, physical and biochemical defenses common to fruit development, and in some genotypes, pathogen-specific, inducible resistance.

The distinctive biology of the cucumber ARR pathosystem and high-resolution probe of the initial period of infection allowed us to tease out transcriptional processes that are unique in resistant tissue, with minimal introduction of artifacts derived from genetic difference between resistant and susceptible materials. The rapid transcriptional defense response as early as $2-4 \mathrm{hpi}$ and subsequent pathogen death in resistant-aged fruit indicate that upstream components in defense signaling, such as pathogen receptors or transcription factors, are developmentally regulated and enable pathogen sensing at the resistant age. In our previous analyses of ARR expressing fruit, we observed developmentally-regulated accumulation of chemical compounds with inhibitory effects on the $P$. capsici growth in vitro $[14,15]$. We further showed a developmental upregulation of four pathogen receptors that was unique to the ARR expressing genotype [15]. Here we observed similar upregulation of specialized metabolism and defense in uninoculated 16 dpp fruit. Together, these earlier and current results suggest a model (Fig. 10) in which accumulated antimicrobial specialized metabolites and an early response to infection mediated by developmentally-regulated expression of receptor-like genes, contribute to ARR. Transcription factors such as WRKY33 are expressed, and their downstream targets including ethylene synthesis genes and other defense genes are activated. In resistant-aged fruit, metabolism of ROS is also rapidly activated, likely further mediating a strong defense.

\section{Conclusion}

In conclusion, our studies indicate that ARR in the cucumber fruit - P. capsici system likely results from a combination of preformed and inducible defenses. Biochemical and other developmentally intrinsic defenses may act in concert with developmentally regulated expression of pathogen recognition-triggered, inducible responses. As production and maintenance of plant defenses is energy intensive and can be costly to fitness, an ideally timed and placed defense, is crucial for plant evolutionary success [9]. Plants expressing ARR optimize these defenses ontogenically, to express them at the opportune physiological age. For cucumber fruit, manifestation of these defenses coincides with the end of exponential fruit growth, protecting the developing seeds for the remaining weeks until the seeds and fruit reach maturity.

\section{Methods}

\section{Plant material}

All experiments were performed in the greenhouse as described in Ref. [21], except with drip fertigation $(1 \mathrm{~L} /$ day at $1-2 \%$ 20-20-20 fertilizer). To identify cultivars that express ARR, a set of 22 inbred cucumber cultivars was grown in the greenhouse, and three to ten fruits were collected from each cultivar at $16 \mathrm{dpp}$ to test for ARR. The source of seeds for these cultivars is included in Supplementary Table 1. Cucumber cv. 'Poinsett 76' (seeds obtained from Seedway, LLC, Hall, NY) was used for all subsequent experiments. In transcriptome experiment 1 , two flowers were hand pollinated per plant, while in transcriptome experiment two flowers were tagged at anthesis and bee pollinated. In both experiments, pollination was staggered, such that 8 and $16 \mathrm{dpp}$ fruit were harvested on the same day. After fruit set only one fruit per plant was retained; any other fruits were removed, to prevent competition. 


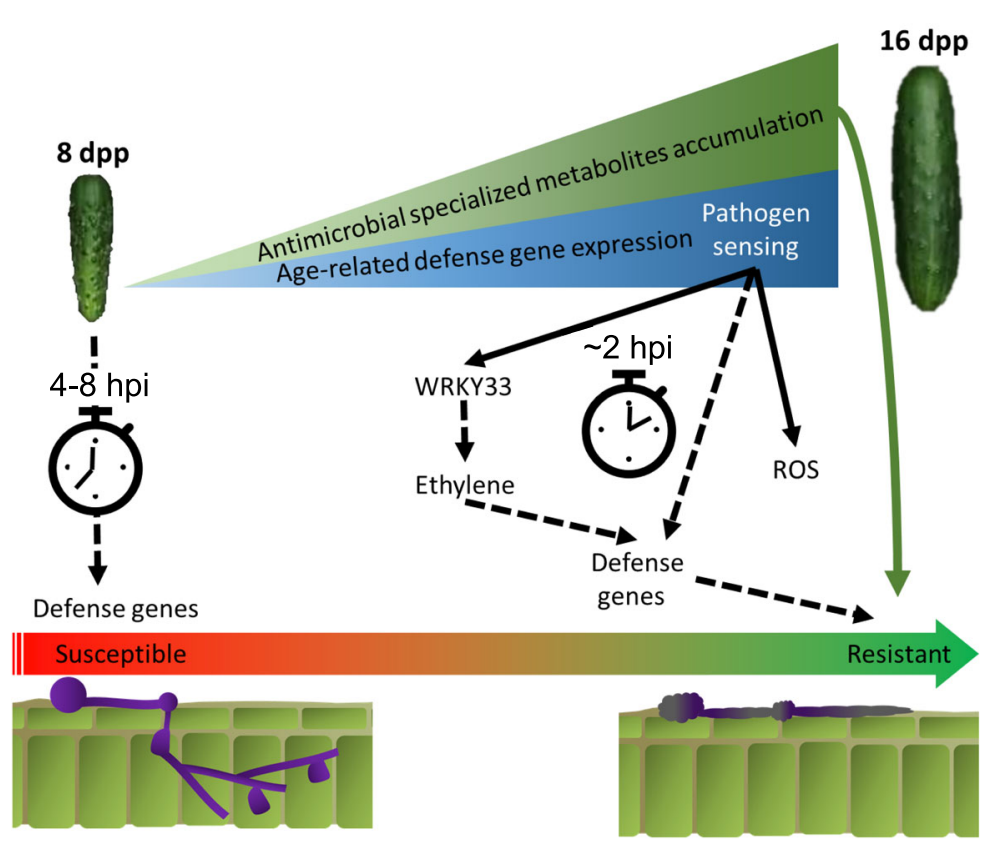

Fig. 10 Hypothesized model for cucumber age-related resistance to $P$. capsici. In young susceptible fruit there is low accumulation of potentially antimicrobial specialized metabolites. Furthermore, a delayed response (> 8 hpi) to pathogen sensing may be too late to limit pathogen establishment. In resistant-aged fruit, the accumulation of metabolites could directly inhibit pathogen growth. Developmentally regulated expression of receptor-like gene(s) allows the sensing of pathogen-associated molecular patterns or effectors, and thus mediates an early response to infection. Transcription factors such as WRKY33 are expressed and their downstream targets including ethylene synthesis genes and other defense genes are activated. In resistant-aged fruit, metabolism of reactive oxygen species (ROS) is also activated in an early response to infection likely further mediating a strong defense response. By $24 \mathrm{hpi}$ pathogen presence is limited on the fruit surface and mostly non-viable spores and hyphae remain

\section{Detached fruit inoculations and sample collection}

Harvested fruit were briefly washed with distilled water and allowed to air-dry. Fruits were placed in incubation trays lined with wet paper towels, to maintain high humidity and covered with clear plastic tops. Zoospore suspensions were prepared from $P$. capsici isolate OP97 or NY-0644-1 expressing RFP [25] cultured on diluted V8 agar media (V8 juice $200 \mathrm{~mL}, \mathrm{CaCO}_{3} 3 \mathrm{~g}$, agar 15 g, distilled water $800 \mathrm{~mL}$ ). After 7 days, the plates were flooded with $10 \mathrm{~mL}$ sterile distilled water to release zoospores. Two $10 \mu \mathrm{l}$ aliquots of zoospore suspension were quantified using a Countess Cell Counter (Invitrogen) and the mean concentration was used for dilution. The suspension was diluted to a concentration of $5 \times 10^{5}$ zoospores $/ \mathrm{mL}$. Fruits were then inoculated with $\sim 6$ (8 dpp fruit) or $\sim 12$ (16 dpp fruit), equally spaced, $30 \mu \mathrm{L}$ droplets of the diluted zoospore suspension. Incubation was performed under constant light at $23-25^{\circ} \mathrm{C}$. For ARR screening development of disease symptoms such as water soaking and mycelial growth on each fruit was monitored daily for 10 days. Fruits were evaluated using a disease rating in scale of $1-9$ ( 1 = no symptom; $9=$ extensive mycelial growth and sporulation).

Plant material was inoculated as described above for two transcriptome experiments; the first included fruit sampled at $0,4,24$, and $48 \mathrm{~h}$ post inoculation (hpi), and the second at $0,2,4,8,12,18$, and $24 \mathrm{hpi}$. In both experiments timepoint 0 was collected at $12: 30 \mathrm{pm}$. At each subsequent timepoint, samples were collected from 6 to 12 inoculation sites per fruit. Samples from a given fruit were pooled to form a biological replicate. Three replicate fruits were sampled for each age at each timepoint. Thus, each timepoint had 3 biological replicates, each comprised of multiple inoculated tissue samples. In experiment 2, at timepoint 0 , the three replicate samples were prepared from a single fruit. Fruits were removed from the incubation chamber and punches were made around each inoculation site using a No. 4 cork borer. Peel discs were subsequently collected by peeling the punched area using a vegetable peeler and immediately frozen in liquid nitrogen and stored at $-80^{\circ} \mathrm{C}$ until RNA extraction. In experiment 2, samples taken from uninoculated parts of the fruit were used as the respective control for each time point.

\section{High-throughput RNA extraction}

Samples were ground using a mortar and pestle in liquid nitrogen. RNA extraction was performed using the MagMAX Plant RNA Isolation Kit protocol (Thermo Fisher) with slight modifications: $100-150 \mathrm{mg}$ of ground tissue were added to $1000 \mu \mathrm{L}$ of lysis buffer. High-throughput RNA extraction, performed in 96-well format on a 
KingFisher Flex Purification System (Thermo Fisher), and assessment of RNA concentration and quality were performed as described in Rett-Cadman et al., 2019 [54]. All samples had a minimum RNA quality score of 8 .

\section{TruSeq and QuantSeq 3'-mRNA library preparation and sequencing}

For the first transcriptome experiment, preparation of TruSeq libraries, subsequent clean-up, quality control and quantification were performed as described in [54]. Two pools of 15 libraries were each loaded onto a lane of an Illumina HiSeq 4000 flow cell and sequenced in a $1 \times 50$ bp single read format. Base calling, demultiplexing, and conversion to FastQ format was as described in [54]. For the second experiment, QuantSeq 3'-mRNA FWD libraries (Lexogen) were prepared by the Cornell University, Institute of Biotechnology, Genomics Facility using the manufacturers guidelines. Quality control and quantification of completed libraries was performed using a combination of Qubit dsDNA HS and Advanced Analytical Fragment Analyzer High Sensitivity DNA assays. The libraries where then loaded on a single Illumina NextSeq500 lane and sequenced in a $1 \times 86$ bp single end format. Base calling was achieved by Illumina RTA v2.4.11 and output of RTA was demultiplexed and converted to FastQ format with Illumina Bcl2fastq v2.18.

\section{Sequencing read preprocessing and quasi-mapping Experiment 1}

Reads were cleaned, and adaptor sequences were removed using Trimmomatic v. 0.34 [55] with the following settings: LEADING:3 TRAILING:3 SLIDINGW INDOW:4:15 MINLEN:35. Quality control was performed using FastQC (http://www.bioinformatics.bbsrc. ac.uk/projects/fastqc). A cucumber transcriptome fasta file was made from the 'Chinese Long' (v2) [56, 57] (ftp://cucurbitgenomics.org/pub/cucurbit/genome/cucumber/Chinese_long/v2/) genome using the gffread function from the cufflinks software package [58] and high-quality reads were then quasi-mapped to the transcriptome using Salmon v. 0.9.1 [59] with default settings.

\section{Experiment 2}

The quality of reads was assessed with FastQC and visualized using multiQC [60]. Subsequently, reads were processed using BBMap [61] with the following settings: $\mathrm{ftl}=12 ; \mathrm{k}=13 ; \quad \mathrm{ktrim}=\mathrm{r} ; \quad$ useshortkmers $=\mathrm{t} ; \operatorname{mink}=5$; qtrim $=\mathrm{r} ; \quad$ trimq $=10 ; \quad$ minlength $=20 ; \quad$ int $=\mathrm{f}, \quad$ and trimmed of any poly A sequences, adaptors and the first $12 \mathrm{nt}$ (as recommended by the manufacturer of the library kit). To increase the mapping success rate, the 'Chinese Long' (v2) GFF3 file (ftp://cucurbitgenomics. org/pub/cucurbit/genome/cucumber/Chinese_long/v2/) was amended to extend all transcript 3'UTRs by 1000 bases using a custom $\mathrm{R}$ script. If a gene model existed prior to 1000 bases, on the same strand, then the 3'UTR was extended only until 1 base before that next gene. The extended gene models were then used to extract a transcriptome fasta file as above. Reads were then quasimapped to this new transcriptome file using Salmon v 0.12.0 with the --noLengthCorrection option. Efficacy of Salmon for mapping 3'RNA reads was benchmarked by comparing to whole genome alignment using BWA [62] and htseq-count [63], with comparable results.

\section{Differential expression analysis}

Read quantification data was imported into $\mathrm{R}$ using the tximport $\mathrm{R}$ package [64] and differential expression analysis was performed using DEseq2 [65] with log-foldchange-shrinkage. Contrasts were analyzed comparing sequential timepoints as well as each timepoint vs. uninoculated samples. DEG were called significant using an adjusted $p$-value (Benjamini-Hochberg adjustment [66]) and a false discovery rate of less than $5 \%$. A cutoff expression change of above two-fold was used to define biological significance. Alluvial plots were drawn using the ggalluvial $\mathrm{R}$ package and venn diagrams were created using the overLapper script from Ref. [67].

\section{Weighted gene co-expression network analysis}

Integer value transcript counts from experiment two, were imported into DESeq2 using the tximport R package [64]. Genes with less than 5 reads in greater than 75 of the total 78 samples were considered lowly expressed and excluded from the analysis, and 15,202 genes remained. The normalized counts matrix was then transformed using the variance stabilizing transformation (VST) [68] using DESeq2 and imported into the WGCNA package pipeline [26].

Two separate signed networks were assigned for the inoculated susceptible- and resistant-aged fruit. For each network, the VST counts were used to calculate adjacency matrices using the biweight midcorrelation and a soft thresholding power of 12 (yielding a scale free topology fit of greater than 0.8). The adjacency matrices were used to calculate two topological overlap dissimilarity matrices which were subsequently used for forming gene clustering trees, using average distances. The gene trees were used for assigning co-expression modules using the dynamic tree cut algorithm with a minimum module size of 30 genes. Module eigengenes were correlated to each other and modules with similar expression patterns (dissimilarity <0.25) were merged. Gene expression profiles of module genes from the infected resistant network (16 dpp) were plotted based on VST values and compared to control and $8 \mathrm{dpp}$ expression patterns. 
To identify modules with different expression patterns in inoculated tissue, read counts were first extracted and normalized by library size using DESeq2 counts() function. For each of the resistant network modules, the $\log _{2}$ $(+0.5)$ of the normalized counts of all genes in that module was the dependent variable in a linear model where a natural cubic spline with 3 internal knots, at 3 , 8,15 hpi (as determined by the $0.25,0.5$ and 0.75 quartiles of time), was applied to the time variable using the $n s()$ function from the splines $\mathrm{R}$ package:

$$
\begin{aligned}
\log _{2}\left(\text { Counts }_{\text {Module }}+0.5\right) & =\text { age }+ \text { age } \\
& : \text { ns }(\text { time }, d f=4)
\end{aligned}
$$

An analysis of variance was then performed to identify module with significant age $\times$ splined-time interactions. The summary of each of those linear models contains the interaction effects for each segment of the spline. Modules with interaction effects in segment 1 and/or 2 (0-3 and 3-8 hpi, respectively) were identified as early induced modules.

\section{Gene ontology term enrichment analysis}

Gene Ontology (GO) term enrichment analysis was performed using the TopGO R package [69] with the entire set of fruit peel expressed genes set as background. Terms were considered enriched if they passed a $p$-value of 0.05 on the Fisher test with the "weight01" algorithm and a minimum node size of 100 genes. The previously updated GO term list for cucumber genes [15], was used for analysis. To visualize change of GO terms over consecutive contrasts heatmaps of $-\log _{10}$ (Fisher weight01 $p$-values) were plotted using only terms with $P<0.01$.

\section{Code availability}

All code and scripts for the entire analysis are available in Supplementary File 5 and at: https:/github.com/ bmansfeld/cucumberPcapInfection

\section{Microscopy}

Preliminary fluorescent microscopy of infection was performed using an EVOS FL Auto imaging system (ThermoFisher). Excised cucumber peels were affixed to the lid of a $100 \mathrm{~mm}$ petri dish using petroleum jelly and inoculated with $10 \mu \mathrm{L}$ zoospore suspension $\left(\sim 5 \times 10^{5}\right.$ spores/ml) of RFP-expressing isolate NY-0664-1 [25]. Petri dishes were then sealed with parafilm and carefully inverted and placed on the microscope table. Samples were observed at 2 and $4 \mathrm{x}$ magnification and images were captured every $30 \mathrm{~min}$ for $72 \mathrm{~h}$. Additional images were taken at 20x magnification at $4 \mathrm{hpi}$.

While collecting inoculated samples for transcriptome experiment 2, a $\sim 2 \mathrm{~mm}$ peel plug from the middle section of each fruit was also excised using a razor blade and fixed in $4 \%$ glutaraldehyde in $0.1 \mathrm{M}$ phosphate buffer for scanning electron microscopy. After overnight fixation in glutaraldehyde, samples were soaked in $0.1 \mathrm{M}$ phosphate buffer for $40 \mathrm{~min}$. After consecutive dehydration in rising ethanol concentrations $(25,50,75,90,100$, $100,100 \%$; $1 \mathrm{~h}$ each), samples were transferred to a Leica Microsystems EM CPD300 critical point dryer (Leica Microsystems) using liquefied carbon dioxide as the transitional fluid. Samples were then mounted on aluminum stubs using adhesive Tabs (M.E. Taylor Engineering) and coated with osmium ( $\sim 10 \mathrm{~nm}$ thickness) in an NEOC-AT osmium coater (Meiwafosis Co., Ltd.). Samples were examined in a JEOL JSM-6610LV scanning electron microscope (JEOL Ltd.).

\section{High-throughput infection phenotyping}

High-throughput in vivo disease phenotyping was performed as described in Ref. [70]. Briefly, sixteen $6 \mathrm{~mm}$ diameter, $5 \mathrm{~mm}$ thick, peel tissue plugs were collected from each of three 8 and $16 \mathrm{dpp}$ fruit using a biopsy punch. Plugs were placed in a 96-well black plate and subsequently inoculated with the constitutively fluorescing P. capsici isolate NY-0664-1 [25], or with distilled water (control - 4 plugs/fruit). Plates were read using a Tecan Spark Plate Reader (Tecan). Fluorescent measurements were taken in each well every hour, over the course of $24 \mathrm{~h}$ at $28^{\circ} \mathrm{C}$. The excitation and emission settings were 536 and $586 \mathrm{~nm}$, respectively. Gain was calculated from a well containing a mycelial mat, and the Zposition was set at $20000 \mu \mathrm{m}$.

\section{Supplementary information}

Supplementary information accompanies this paper at https://doi.org/10. 1186/s12864-020-07040-9.

Additional file 1: Supplementary file 1.: Genes involved in an early incompatible interaction

Additional file 2: Supplementary file 2: Gene module assignment and normalized expression - resistant network

Additional file 3: Supplementary file 3. Gene module assignment and normalized expression - susceptible network

Additional file 4: Supplementary file 4. GO-term enrichment analysis of the resistant network modules

Additional file 5: Supplementary file 5. All code and analysis Additional file 6 .

\section{Abbreviations}

ARR: Age-related resistance; DEG: Differentially expressed genes; dpp: Days post-pollination; GO: Gene ontology; hpi: Hours post-inoculation; ROS: Reactive oxygen species; SEM: Scanning electron microscopy; VST: Variance stabilizing transformation; PCA: Principal component analysis; WGCNA: Weighted gene co-expression network analysis

\section{Acknowledgments}

We thank the Sue Hammar for greenhouse and experiment assistance, and the Michigan State University (MSU) Research Technology Support Facility Genomics Core and Cornell University, Institute of Biotechnology, Genomics 
Facility, for library preparation and sequencing. We thank the MSU Center for Advanced Microscopy for scanning electron microscopy work. We also acknowledge the Michigan State University Research Center for Statistical Training and Consulting. Finally, we thank Drs. Cornelius Barry, Robin Buell, Brad Day, Sheng Yang He and Robert VanBuren for critical reading of the manuscript.

\section{Authors' contributions}

BNM and RG conceived of the research and wrote the manuscript. BNM performed the RNA-seq experiments, transcriptome and network analyses. MC screened the cultivar collection for ARR and performed the developmental phenotyping for ARR in 'Poinsett 76. CZ and BNM developed and performed the in vivo bioassay. $Y L$ and BNM performed the fluorescent microscopy experiments. All authors have read and approved the manuscript.

\section{Funding}

This work was in part supported by the National Institute of Food and Agriculture, U.S. Department of Agriculture, under award number 2015-51181-24285, MSU Project GREEEN, and by USDA NIFA Hatch project number MICL02647 to RG. CZ was supported by the China Scholarship Council. The funders had no role in study design, data collection and analysis, decision to publish, or preparation of the manuscript.

\section{Availability of data and materials}

Raw reads for both experiments are deposited in the NCBI Sequence Read Archive (SRA) database under the accession number PRJNA575868.

\section{Ethics approval and consent to participate}

Not applicable.

\section{Consent for publication}

Not applicable.

\section{Competing interests}

The authors declare that they have no competing interests.

\section{Author details}

${ }^{1}$ Graduate Program in Plant Breeding, Genetics and Biotechnology and Department of Horticulture, Michigan State University, 1066 Bogue St, East Lansing, Ml 48824, USA. ${ }^{2}$ Key Laboratory of Biology and Genetic Improvement of Horticultural Crops (North China), Beijing Key Laboratory of Vegetable Germplasm Improvement, National Engineering Research Center for Vegetables, Beijing Academy of Agriculture and Forestry Sciences, Beijing, China.

\section{Received: 10 January 2020 Accepted: 31 August 2020}

\section{Published online: 11 September 2020}

\section{References}

1. Whalen MC. Host defence in a developmental context. Mol Plant Pathol. 2005;6:347-60

2. Develey-Rivière M-P, Galiana E. Resistance to pathogens and host developmental stage: a multifaceted relationship within the plant kingdom. New Phytol. 2007;175:405-16. https://doi.org/10.1111/j.1469-8137.2007. 02130.x.

3. Hu L, Yang L. Time to fight: Molecular mechanisms of age-related resistance. Phytopathology. 2019;109:1-28.

4. Kim YJ, Hwang BK, Park KW. Expression of age-related resistance in pepper plants infected with Phytophthora capsici. Plant Dis. 1989;73:745-7.

5. Gee CT, Gadoury DM, Cadle-Davidson L. Ontogenic resistance to Uncinula necator varies by genotype and tissue type in a diverse collection of Vitis spp. Plant Dis. 2008;92:1067-73. https://doi.org/10.1094/PDIS-92-7-1067.

6. Ando K, Hammar S, Grumet R. Age-related resistance of diverse cucurbit fruit to infection by Phytophthora capsici. J Amer Soc Hort Sci. 2009;134: 176-82 http://journal.ashspublications.org/content/134/2/176.short. Accessed 10 Jul 2014.

7. Zhao J, Fu J, Li X, Xu C, Wang S. Dissection of the factors affecting development-controlled and race-specific disease resistance conferred by leucine-rich repeat receptor kinase-type $\mathrm{R}$ genes in rice. Theor Appl Genet. 2009;119:231-9. https://doi.org/10.1007/s00122-009-1032-3.
8. Zhang H, Wang C, Cheng Y, Chen X, Han Q, Huang L, et al. Histological and cytological characterization of adult plant resistance to wheat stripe rust. Plant Cell Rep. 2012;31:2121-37. https://doi.org/10.1007/s00299-012-1322-0.

9. Meldau S, Erb M, Baldwin IT. Defence on demand: mechanisms behind optimal defence patterns. Ann Bot. 2012;110:1503-14.

10. Alzohairy SA, Hammerschmidt R, Hausbeck MK. Changes in winter squash fruit exocarp structure associated with age-related resistance to Phytophthora capsici. Phytopathology. 2020;1 10:447-55. https://doi.org/10.1094/PHYTO-04-19-0128-R.

11. Cao $Y$, Ding $X$, Cai M, Zhao J, Lin Y, Li X, et al. The expression pattern of a rice disease resistance gene $\mathrm{Xa3} / \mathrm{Xa26}$ is differentially regulated by the genetic backgrounds and developmental stages that influence its function. Genetics. 2007;177:523-33.

12. Zou Y, Wang S, Zhou Y, Bai J, Huang G, Liu X, et al. Transcriptional regulation of the immune receptor FLS2 controls the ontogeny of plant innate immunity. Plant Cell. 2018;30 November:tpc.00297.2018. https://doi. org/10.1105/tpc.18.00297.

13. Gevens AJ, Ando K, Lamour KH, Grumet R, Hausbeck MK. A detached cucumber fruit method to screen for resistance to Phytophthora capsici and effect of fruit age on susceptibility to infection. Plant Dis. 2006;90:1276-82. https://doi.org/10.1094/PD-90-1276.

14. Ando K, Carr KM, Colle M, Mansfeld BN, Grumet R. Exocarp properties and transcriptomic analysis of cucumber (Cucumis sativus) fruit expressing agerelated resistance to Phytophthora capsici. PLoS One. 2015;10:e0142133. https://doi.org/10.1371/journal.pone.0142133.

15. Mansfeld BN, Colle M, Kang Y, Jones AD, Grumet R. Transcriptomic and metabolomic analyses of cucumber fruit peels reveal a developmental increase in terpenoid glycosides associated with age-related resistance to Phytophthora capsici. Hortic Res. 2017;4 February:17022. https://doi.org/10.1038/hortres.2017.22.

16. Hausbeck MK, Lamour KH. Phytophthora capsici on vegetable crops: research progress and management challenges. Plant Dis. 2004;88:1292-303.

17. Granke LL, Quesada-Ocampo L, Lamour K, Hausbeck MK. Advances in research on Phytophthora capsici on vegetable crops in the United States. Plant Dis. 2012;96:1588-600. https://doi.org/10.1094/PDIS-02-12-0211-FE.

18. Lamour KH, Stam R, Jupe J, Huitema E. The oomycete broad-host-range pathogen Phytophthora capsici. Mol Plant Pathol. 2012;13:329-37. https:// doi.org/10.1111/J.1364-3703.2011.00754.X.

19. Jupe J, Stam R, Howden AJ, Morris JA, Zhang R, Hedley PE, et al. Phytophthora capsici-tomato interaction features dramatic shifts in gene expression associated with a hemi-biotrophic lifestyle. Genome Biol. 2013; 14:R63. https://doi.org/10.1186/gb-2013-14-6-r63.

20. Colle M, Straley E, Makela SB, Hammar SA, Grumet R. Screening the cucumber plant introduction collection for young fruit resistance to Phytophthora capsici. HortScience. 2014;49:244-9.

21. Ando K, Carr KM, Grumet R. Transcriptome analyses of early cucumber fruit growth identifies distinct gene modules associated with phases of development BMC Genomics. 2012;13:518-34. https://doi.org/10.1186/1471-2164-13-518.

22. Huot B, Yao J, Montgomery BL, He SY. Growth-defense tradeoffs in plants: a balancing act to optimize fitness. Mol Plant. 2014;7:1267-87. https://doi.org/ $10.1093 / \mathrm{mp} /$ ssu049.

23. Biles CL. Relationship of Phytophthora fruit rot to fruit maturation and cuticle thickness of new Mexican-type peppers. Phytopathology. 1993;83: 607. https://doi.org/10.1094/phyto-83-607.

24. Gusberti M, Gessler C, Broggini GAL. RNA-seq analysis reveals candidate genes for ontogenic resistance in Malus-Venturia Pathosystem. PLoS One. 2013:8:e78457. https://doi.org/10.1371/journal.pone.0078457.

25. Dunn AR, Fry BA, Lee TY, Conley KD, Balaji V, Fry WE, et al. Transformation of Phytophthora capsici with genes for green and red fluorescent protein for use in visualizing plant-pathogen interactions. Australas Plant Pathol. 2013; 42:583-93. https://doi.org/10.1007/s13313-013-0222-2.

26. Langfelder P, Horvath S. WGCNA: an R package for weighted correlation network analysis. BMC Bioinformatics. 2008;9:559. https://doi.org/10.1186/ 1471-2105-9-559.

27. Langfelder $\mathrm{P}$, Luo R, Oldham MC, Horvath S. Is my network module preserved and reproducible? PLoS Comput Biol. 2011;7:e1001057. https:// doi.org/10.1371/journal.pcbi.1001057.

28. Erwin DC, Ribeiro OK. Phytophthora diseases worldwide. St. Paul: American Phytopathological Society (APS Press); 1998.

29. Nowicki M, Foolad MR, Nowakowska M, Kozik EU. Potato and tomato late blight caused by Phytophthora infestans: an overview of pathology and resistance breeding. Plant Dis. 2012;96:4-17. https://doi.org/10.1094/PDIS-0511-0458. 
30. Gyetvai G, Sønderkær M, Göbel U, Basekow R, Ballvora A, Imhoff M, et al. The transcriptome of compatible and incompatible interactions of potato (Solanum tuberosum) with Phytophthora infestans revealed by DeepSAGE analysis. PLoS One. 2012;7:e31526.

31. Zuluaga AP, Vega-Arreguín JC, Fei Z, Matas AJ, Patev S, Fry WE, et al. Analysis of the tomato leaf transcriptome during successive hemibiotrophic stages of a compatible interaction with the oomycete pathogen Phytophthora infestans. Mol Plant Pathol. 2016;17:42-54.

32. Oh E. Resistance mechanisms of port-orford-cedar to Phytophthora lateralis. Ph.D. Dissertation. Corvallis: Oregon State University; 2004. p. 207.

33. Malajczuk N. Interaction between Phytophthora cinnamomi zoospores and micro-organisms on non-mycorrhizal and ectomycorrhizal roots of Eucalyptus marginata. Trans Br Mycol Soc. 1988;90:375-82. https://doi.org/10. 1016/S0007-1536(88)80146-3.

34. Soylu EM, Soylu S, Kurt S. Antimicrobial activities of the essential oils of various plants against tomato late blight disease agent Phytophthora infestans. Mycopathologia. 2006;161:119-28.

35. Khan MA, Zhihui C. Influence of garlic root exudates on cyto-morphological alteration of the hyphae of Phytophthora capsici, the cause of Phytophthora blight in pepper. Pakistan J Bot. 2010;42:4353-61.

36. Khan MA, Zhihui $C$, Xuemei $X$, Khan AR, Ahmed SS. Ultrastructural studies of the inhibition effect against Phytophthora capsici of root exudates collected from two garlic cultivars along with their qualitative analysis. Crop Prot. 2011;30:1149-55. https://doi.org/10.1016/j.cropro.2011.04.013.

37. de Beer A, Vivier MA. Four plant defensins from an indigenous south African Brassicaceae species display divergent activities against two test pathogens despite high sequence similarity in the encoding genes. BMC Res Notes. 2011;4:459. https://doi.org/10.1186/1756-0500-4-459.

38. Sagaram US, Pandurangi R, Kaur J, Smith TJ, Shah DM. Structure-activity determinants in antifungal plant defensins MsDef1 and MtDef4 with different modes of action against Fusarium graminearum. PLoS One. 2011;6: 18550. https://doi.org/10.1371/journal.pone.0018550.

39. Windram O, Madhou P, McHattie S, Hill C, Hickman R, Cooke E, et al. Arabidopsis defense against Botrytis cinerea: chronology and regulation deciphered by high-resolution temporal transcriptomic analysis. Plant Cell. 2012;24:3530-57. https://doi.org/10.1105/tpc.112.102046.

40. Wang Y, Bouwmeester K, van de Mortel JE, Shan W, Govers F. A novel Arabidopsis-oomycete pathosystem: differential interactions with Phytophthora capsici reveal a role for camalexin, indole glucosinolates and salicylic acid in defence. Plant Cell Environ. 2013;36:1192-203.

41. Mine A, Seyfferth C, Kracher B, Berens ML, Becker D, Tsuda K. The defense phytohormone signaling network enables rapid, high-amplitude transcriptional reprogramming during effector-triggered immunity. Plant Cell. 2018:30:1199-219. https://doi.org/10.1105/tpc.17.00970.

42. Tang D, Wang G, Zhou J-M. Receptor kinases in plant-pathogen interactions: more than pattern recognition. Plant Cell. 2017;29:618-37. https://doi.org/10.1105/tpc.16.00891.

43. Devaiah BN, Karthikeyan AS, Raghothama KG. WRKY75 transcription factor is a modulator of phosphate acquisition and root development in Arabidopsis. Plant Physiol. 2007;143:1789-801.

44. Zheng Z, Qamar SA, Chen Z, Mengiste T. Arabidopsis WRKY33 transcription factor is required for resistance to necrotrophic fungal pathogens. Plant J. 2006;48:592-605.

45. Birkenbihl RP, Diezel C, Somssich IE. Arabidopsis WRKY33 is a key transcriptional regulator of hormonal and metabolic responses toward Botrytis cinerea infection. Plant Physiol. 2012;159:266-85.

46. Birkenbihl RP, Kracher B, Roccaro M, Somssich IE. Induced genome-wide binding of three Arabidopsis WRKY transcription factors during early MAMPtriggered immunity. Plant Cell. 2016;29:20-38.

47. Howden AJM, Stam R, Martinez Heredia V, Motion GB, ten Have S, Hodge K, et al. Quantitative analysis of the tomato nuclear proteome during Phytophthora capsici infection unveils regulators of immunity. New Phytol. 2017;215:309-22. https://doi.org/10.1111/nph.14540.

48. Broekgaarden C, Vos IA, Pieterse CMJ, Van Wees SCM, Caarls L. Ethylene: traffic controller on hormonal crossroads to defense. Plant Physiol. 2015;169: 2371-9.

49. Núñez-Pastrana R, Arcos-Ortega GF, Souza-Perera RA, Sánchez-Borges CA, Nakazawa-Ueji YE, García-Villalobos FJ, et al. Ethylene, but not salicylic acid or methyl jasmonate, induces a resistance response against Phytophthora capsici in habanero pepper. Eur J Plant Pathol. 2011;131:669-83. https://doi. org/10.1007/s10658-011-9841-z.
50. Shibata Y, Kawakita K, Takemoto D. Age-related resistance of Nicotiana benthamiana against hemibiotrophic pathogen Phytophthora infestans requires both ethylene- and salicylic acid-mediated signaling pathways. Mol PlantMicrobe Interact. 2010;23:1130-42. https://doi.org/10.1094/MPMI-23-9-1130.

51. Quan $\sqcup$, Zhang B, Shi WW, Li HY. Hydrogen peroxide in plants: a versatile molecule of the reactive oxygen species network. J Integr Plant Biol. 2008; 50:2-18.

52. Li Y, Pennington $\mathrm{BO}$, Hua J. Multiple R-like genes are negatively regulated by BON1 and BON3 in Arabidopsis. Mol Plant-Microbe Interact. 2009;22:840-8.

53. Gou M, Zhang Z, Zhang N, Huang Q, Monaghan J, Yang H, et al. Opposing effects on two phases of defense responses from concerted actions of HEAT SHOCK COGNATE70 and BONZAI1 in Arabidopsis. Plant Physiol. 2015; 169:2304-23.

54. Rett-Cadman S, Colle M, Mansfeld B, Barry CS, Wang Y, Gao L, Fei Z, Grumet R. QTL and transcriptomic analyses implicate cuticle transcription factor SHINE as a source of natural variation for epidermal traits in cucumber fruit. Front Plant Sci. 2019;01536. doi.org/https://doi.org/10.3389/fpls.2019.01536.

55. Bolger AM, Lohse M, Usadel B. Trimmomatic: a flexible trimmer for Illumina sequence data. Bioinformatics. 2014;30:2114-20.

56. Huang S, Li R, Zhang Z, Li L, Gu X, Fan W, et al. The genome of the cucumber, Cucumis sativus L. Nat Genet. 2009;41:1275-81.

57. Li Z, Zhang Z, Yan P, Huang S, Fei Z, Lin K. RNA-Seq improves annotation of protein-coding genes in the cucumber genome. BMC Genomics. 2011;12: 540-51. https://doi.org/10.1186/1471-2164-12-540.

58. Trapnell C, Williams BA, Pertea G, Mortazavi A, Kwan G, van Baren MJ, et al. Transcript assembly and quantification by RNA-Seq reveals unannotated transcripts and isoform switching during cell differentiation. Nat Biotechnol. 2010;28:511-5. https://doi.org/10.1038/nbt.1621.

59. Patro R, Duggal G, Love MI, Irizarry RA, Kingsford C. Salmon provides fast and bias-aware quantification of transcript expression. Nat Methods. 2017; 14:417-9. https://doi.org/10.1038/nmeth.4197.

60. Ewels P, Magnusson M, Lundin S, Käller M. MultiQC: summarize analysis results for multiple tools and samples in a single report. Bioinformatics. 2016;32:3047-8.

61. JGI Joint Genome Institute. BBTools. https://jgi.doe.gov/data-and-tools/ bbtools/. Accessed 28 Mar 2020.

62. Li H, Durbin R. Fast and accurate short read alignment with burrowswheeler transform. Bioinformatics. 2009;25:1754-60.

63. Anders S, Pyl PTP, Huber W. HTSeq-A Python framework to work with highthroughput sequencing data. Bioinformatics. 2015;31:166-9. https://doi.org/ 10.1093/bioinformatics/btu638.

64. Soneson C, Love MI, Robinson MD. Differential analyses for RNA-seq: transcript-level estimates improve gene-level inferences. F1000Research. 2015;4:1521. https://doi.org/10.12688/f1000research.7563.1.

65. Love Ml, Huber W, Anders S. Moderated estimation of fold change and dispersion for RNA-seq data with DESeq2. Genome Biol. 2014;15:1-34.

66. Benjamini $Y$, Hochberg $Y$. Controlling the false discovery rate: a practical and powerful approach to multiple testing. J Royal Stat Soc B. 1995;57:289300. https://doi.org/10.2307/2346101.

67. Girke T. R Script. http://faculty.ucr.edu/ tgirke/Documents/R_BioCond/My_ R_Scripts/overLapper.R.

68. Anders S, Huber W. Differential expression analysis for sequence count data. Genome Biol. 2010;11:R106. https://doi.org/10.1186/gb-2010-11-10-r106.

69. Alexa A, Rahnenfuhrer J, Lengauer T. Improved scoring of functional groups from gene expression data by decorrelating GO graph structure. Bioinformatics. 2006;22:1600-7. https://doi.org/10.1093/bioinformatics/ btl140.

70. Zhang C, Mansfeld BN, Grumet R. Development of a real-time fluorescencebased microplate assays for pathogen growth on plant tissue: Phytophthora capsici infection of cucumber fruit. In: Cucurbitaceae 2018. 2018.

\section{Publisher's Note}

Springer Nature remains neutral with regard to jurisdictional claims in published maps and institutional affiliations. 